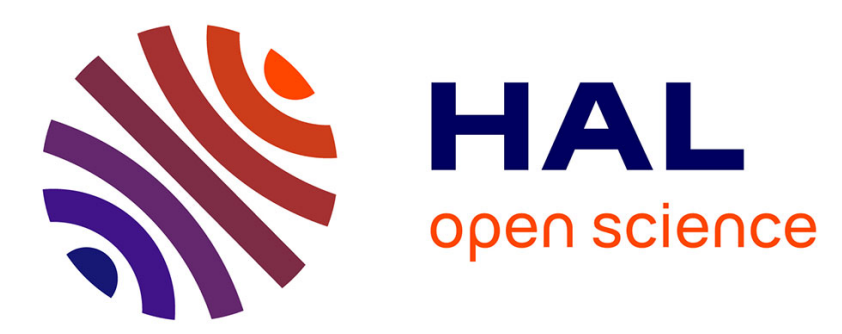

\title{
No-gap Second-order Optimality Conditions for Optimal Control Problems with a Single State Constraint and Control
}

\author{
J. Frederic Bonnans, Audrey Hermant
}

\section{- To cite this version:}

J. Frederic Bonnans, Audrey Hermant. No-gap Second-order Optimality Conditions for Optimal Control Problems with a Single State Constraint and Control. [Research Report] RR-5837, INRIA. 2006, pp.30. inria-00070189

\section{HAL Id: inria-00070189 \\ https://hal.inria.fr/inria-00070189}

Submitted on 19 May 2006

HAL is a multi-disciplinary open access archive for the deposit and dissemination of scientific research documents, whether they are published or not. The documents may come from teaching and research institutions in France or abroad, or from public or private research centers.
L'archive ouverte pluridisciplinaire HAL, est destinée au dépôt et à la diffusion de documents scientifiques de niveau recherche, publiés ou non, émanant des établissements d'enseignement et de recherche français ou étrangers, des laboratoires publics ou privés. 
INSTITUT NATIONAL DE RECHERCHE EN INFORMATIQUE ET EN AUTOMATIQUE

\section{No-gap Second-order Optimality Conditions for Optimal Control Problems with a Single State Constraint and Control}

Frédéric Bonnans — Audrey Hermant

\section{$\mathbf{N}^{\circ} \mathbf{5 8 3 7}$}

February 2006

Thème NUM

\section{apport}

de recherche 



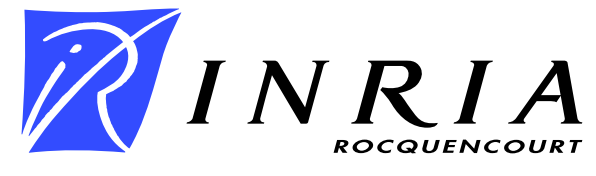

\title{
No-gap Second-order Optimality Conditions for Optimal Control Problems with a Single State Constraint and Control
}

\author{
Frédéric Bonnans国，Audrey Hermant 固 \\ Thème NUM — Systèmes numériques \\ Projet SYDOCO \\ Rapport de recherche $\mathrm{n}^{\circ} 5837$ - February 2006 - 30 pages
}

\begin{abstract}
The paper deals with optimal control problems with only one control variable and one state constraint, of arbitrary order. We consider the case of finitely many boundary arcs and touch times. We obtain a no-gap theory of second-order conditions, allowing to characterize second-order quadratic growth.
\end{abstract}

Key-words: Optimal control, second-order optimality conditions, state constraint, quadratic growth, regular junctions.

* frederic.bonnans@inria.fr

$\dagger$ INRIA, BP 105, 78153 Le Chesnay, France

$\ddagger$ audrey.hermant@inria.fr 


\section{Conditions d'optimalité nécessaires et suffisantes du second ordre pour les problèmes de commande optimale avec un contrôle scalaire et une contrainte sur l'état}

Résumé : Dans cet article, nous étudions un problème de commande optimale avec un contrôle scalaire et une contrainte sur l'état d'ordre quelconque. Les instants de jonctions sont supposés en nombre fini. Nous obtenons des conditions d'optimalité du second ordre nécessaires et suffisantes, qui permettent de caractériser la croissance quadratique.

Mots-clés : Commande optimale, conditions d'optimalité du second ordre, contrainte sur l'état, croissance quadratique, jonctions régulières. 


\section{Introduction}

Considerable efforts have been done in the past for reducing the gap between second-order necessary or sufficient optimality conditions for optimization problems in Banach spaces, with so-called cone constraint (i.e. the constraint mapping must be in a convex cone, or more generally in a convex set). This framework includes many optimal control problems. The theory of second-order necessary optimality conditions involves a term taking into account the curvature of the convex set, see Kawasaki [13, Cominetti [6]. By contrast, second-order sufficient optimality conditions typically involve no such term; see e.g. Maurer and Zowe [21]. We say that a no-gap condition holds, when the only change between necessary or sufficient second-order optimality conditions is between a strict and non strict inequality. In that case it is usually possible to obtain a characterization of the second-order growth condition. There are essentially two cases when no-gap conditions were obtained: (i) the polyedric framework, in the case when the Hessian of Lagrangian is a Legendre form, originating in the work by Haraux [8] and Mignot [22, applied to optimal control problems in e.g. Sokolowski [27] and Bonnans [1, and the extended polyhedricity framework in [4, Section 3.2.3]; this framework essentially covers the case of control constraints (and finitely many final state constraints); and (ii) the second-order regularity framework, introduced in [3] and [2], with applications to semi definite optimization. We refer to [4] for an overview of these theories. No-gap second-order conditions were obtained by Zeidan in [28] for mixed control-state constraints, using conjugate point theory and Ricatti equations.

Generally speaking, problems with non positivity constraints in spaces of continuous functions do not fit into these frameworks. The expression of the curvature term in this case was obtained by Kawasaki [15, 14 in the one dimensional case, and generalized in Cominetti and Penot [7]. However, only sufficient conditions without curvature terms were known. Two exceptions are a quite specific situation studied in [3] (with applications to some eigenvalue problems), and the case of finitely many contact points, when the problem can be reduced locally to finitely many inequality constraints, see e.g. Hettich and Jongen [9].

Our main result is the following. By a localization argument, we split the curvature term into a finite number of contributions of boundary arcs and touch points. Using the theory of junction conditions in Jacobson et al. [12] and Maurer [19], we are able to prove that, under quite weak assumptions, the contribution of boundary arcs to the curvature term is zero. For touch points, we use a reduction argument for those that are essential (i.e. that belong to the support of the multiplier) and we make no hypotheses for the non essential ones. The only delicate point is to compute the expansion of the minimum value of a function in $W^{2, \infty}$. Since it is not difficult to state sufficient conditions taking into account essential reducible touch points, we obtain in this way no-gap conditions, that in addition characterize quadratic growth in a convenient two-norms setting.

The paper is organized as follows. In section 2, we recall the material needed, in both points of view of abstract optimization and junctions conditions analysis. The main contributions of the paper are in sections 35 where the no-gap second-order condition is established. Section 3 states the second-order necessary condition (computation of the curvature term).

RR $\mathrm{n}^{\circ} 5837$ 
Section 4 handles the second-order sufficient condition. In section [5] a reduction approach is presented in order to deal with the non-zero part of the curvature term.

\section{Framework}

We consider the following optimal control problem with a scalar state constraint and a scalar control:

$$
\begin{array}{cc}
(\mathcal{P}) & \min _{u, y} \int_{0}^{T} \ell(u(t), y(t)) \mathrm{d} t+\phi(y(T)) \\
\text { s.t. } & \dot{y}(t)=f(u(t), y(t)) \quad \text { a.e. } t \in[0, T] ; y(0)=y_{0} \\
& g(y(t)) \leq 0 \quad \forall t \in[0, T] .
\end{array}
$$

The data of the problem are the distributed cost $\ell: \mathbb{R} \times \mathbb{R}^{n} \rightarrow \mathbb{R}$, the final cost $\phi: \mathbb{R}^{n} \rightarrow \mathbb{R}$, the dynamics $f: \mathbb{R} \times \mathbb{R}^{n} \rightarrow \mathbb{R}^{n}$, the state constraint $g: \mathbb{R}^{n} \rightarrow \mathbb{R}$, the final time $T>0$, and the initial condition $y_{0} \in \mathbb{R}^{n}$. We make the following assumptions on the data:

(A0) The mappings $\ell, \phi, f$ and $g$ are $k$-times continuously differentiable $\left(C^{k}\right)$ with $k \geq 2$ and have locally Lipschitz continuous second-order derivatives, and the dynamics $f$ is Lipschitz continuous.

(A1) The initial condition satisfies $g\left(y_{0}\right)<0$.

Throughout the paper, it is assumed that assumption (A0) holds.

\subsection{Abstract Optimization}

For $1 \leq p \leq \infty, L^{p}(0, T)$ denotes the Banach space of functions such that

$$
\|u\|_{p}:=\left(\int_{0}^{T}|u(t)|^{p} \mathrm{~d} t\right)^{1 / p}<\infty \text { for } p<\infty ; \quad\|u\|_{\infty}:=\operatorname{supess}|u(t)|<\infty,
$$

and $W^{1, p}(0, T)$ denotes the Sobolev space of functions having a weak derivative in $L^{p}$. The space of continuous functions over $[0, T]$ is denoted by $C[0, T]$, with the norm $\|x\|_{\infty}=$ $\sup |x(t)|$.

Denote by $\mathcal{U}:=L^{\infty}(0, T ; \mathbb{R})\left(\right.$ resp. $\left.\mathcal{Y}:=W^{1, \infty}\left(0, T ; \mathbb{R}^{n}\right)\right)$ the control (resp. state) space. A trajectory is an element $(u, y) \in \mathcal{U} \times \mathcal{Y}$ satisfying the state equation (2). Given $u \in \mathcal{U}$, denote by $y_{u} \in \mathcal{Y}$ the (unique) solution of (2). Under assumption (A0), by the CauchyLipschitz Theorem, this mapping is well-defined and of class $C^{k}$. We may write problem $(\mathcal{P})$ as:

$$
\min _{u \in \mathcal{U}} J(u) \quad ; \quad G(u) \in K
$$


where $J: \mathcal{U} \rightarrow \mathbb{R}$ and $G: \mathcal{U} \rightarrow C[0, T]$ are defined, respectively, by

$$
J(u)=\int_{0}^{T} \ell\left(u(t), y_{u}(t)\right) \mathrm{d} t+\phi\left(y_{u}(T)\right) \quad ; \quad G(u)=g\left(y_{u}\right) .
$$

These mappings are $C^{k}$. Here $K=C_{-}[0, T]$ is the set of continuous functions over $[0, T]$, with values in $\mathbb{R}_{-}$.

We say that $u \in \mathcal{U}$ is a (weak) local optimal solution of (4) that satisfies the quadratic growth condition, if there exists $\alpha>0$ and $\rho>0$ such that:

$$
J(\tilde{u}) \geq J(u)+\alpha\|\tilde{u}-u\|_{2}^{2} \quad \text { for all } \tilde{u} \in B_{\infty}(u, \rho),
$$

where $B_{\infty}(u, \rho)$ denotes the open ball in $L^{\infty}(0, T)$ with center $u$ and radius $\rho$. This condition involves two norms, $L^{\infty}(0, T)$ for the neighborhood, and $L^{2}(0, T)$ for the growth condition.

The space of row vectors is denoted by $\mathbb{R}^{n *}$. The space of Radon measures, dual space to $C[0, T]$, is denoted by $\mathcal{M}[0, T]$ and identified with functions of bounded variation vanishing at zero. The cone of nonnegative measures is denoted by $\mathcal{M}_{+}[0, T]$ and is equal to $K^{-}$, the polar cone of $K$. The duality product over $\mathcal{M}[0, T] \times C[0, T]$ is denoted by $\langle\eta, x\rangle=$ $\int_{0}^{T} x(t) \mathrm{d} \eta(t)$. Adjoint operators (and transpose in $\mathbb{R}^{n}$ ) are denoted by a star ${ }^{*}$. Fréchet derivatives of $f$, etc. w.r.t. arguments $u \in \mathbb{R}, y \in \mathbb{R}^{n}$, are denoted by a subscript, for instance $f_{u}(u, y)=D_{u} f(u, y), f_{u u}(u, y)=D_{u u}^{2} f(u, y)$, etc.

Define the classical Hamiltonian and Lagrangian functions of problem $(\mathcal{P})$, respectively $H: \mathbb{R} \times \mathbb{R}^{n} \times \mathbb{R}^{n *} \rightarrow \mathbb{R}$ and $L: \mathcal{U} \times \mathcal{M}[0, T] \rightarrow \mathbb{R}$ by:

$$
H(u, y, p):=\ell(u, y)+p f(u, y) \quad ; \quad L(u, \eta):=J(u)+\langle\eta, G(u)\rangle .
$$

Denote by $B V(0, T)$ the space of functions of bounded variation. Given $u \in \mathcal{U}$ and $\eta \in$ $\mathcal{M}_{+}[0, T]$, let the costate $p_{u, \eta}$ be the unique solution in $B V\left(0, T ; \mathbb{R}^{n *}\right)$ of:

$$
-\mathrm{d} p_{u, \eta}=\left(\ell_{y}\left(u, y_{u}\right)+p_{u, \eta} f_{y}\left(u, y_{u}\right)\right) \mathrm{d} t+g_{y}\left(y_{u}\right) \mathrm{d} \eta ; \quad p_{u, \eta}(T)=\phi_{y}\left(y_{u}(T)\right) .
$$

Given $v \in \mathcal{U}$, let the linearized state $z_{u, v} \in \mathcal{Y}$ be solution of:

$$
\dot{z}_{u, v}=f_{y}\left(u, y_{u}\right) z_{u, v}+f_{u}\left(u, y_{u}\right) v \quad ; \quad z_{u, v}(0)=0 .
$$

The mapping $\mathcal{U} \rightarrow \mathcal{Y}, v \mapsto z_{u, v}$ is the Fréchet derivative of the mapping $u \mapsto y_{u}$ at point $u$.

The next lemma gives the expressions of derivatives of Lagrangian, with respect to the control. For simplicity of notation, we write in the sequel $D^{2} H_{(u, y)^{2}}(u, y, p)(v, z)^{2}$ instead of $D_{(u, y),(u, y)}^{2} H(u, y, p)((v, z),(v, z))$.

Lemma 2.1. Let $\eta \in \mathcal{M}_{+}[0, T]$. Then $u \mapsto L(u, \eta)$ is of class $C^{2}$ over $\mathcal{U}$, with first and second derivatives given by, for all $v \in \mathcal{U}$ (omitting time argument):

$$
D_{u} L(u, \eta) v=\int_{0}^{T} H_{u}\left(u, y_{u}, p_{u, \eta}\right) v \mathrm{~d} t,
$$




$$
\begin{aligned}
& D_{u u}^{2} L(u, \eta)(v, v)=\int_{0}^{T} D^{2} H_{(u, y)^{2}}\left(u, y_{u}, p_{u, \eta}\right)\left(v, z_{u, v}\right)^{2} \mathrm{~d} t \\
& \quad+z_{u, v}(T)^{*} \phi_{y y}\left(y_{u}(T)\right) z_{u, v}(T)+\int_{0}^{T} z_{u, v}^{*} g_{y y}\left(y_{u}\right) z_{u, v} \mathrm{~d} \eta,
\end{aligned}
$$

where $H$ is given by (6), $z_{u, v}$ and $p_{u, \eta}$ are solution respectively of (8) and (7).

Proof. Since $u \mapsto y_{u}$ is $C^{2}$, the Cauchy-Lipschitz Theorem ensures the existence of the second-order expansion of the state

$$
y_{u+v}=y_{u}+z_{u, v}+\frac{1}{2} z_{u, v v}+o\left(\|v\|_{\infty}^{2}\right) .
$$

It is easily seen, substituting (11) into the state equation and keeping the terms of secondorder, that $z_{u, v v}$ is solution of:

$$
\dot{z}_{u, v v}=f_{y}\left(u, y_{u}\right) z_{u, v v}+D^{2} f_{(u, y)^{2}}\left(u, y_{u}\right)\left(v, z_{u, v}\right)^{2} \quad ; \quad z_{u, v v}(0)=0 .
$$

Using costate equation (7) and linearized state equations (8) and (12), we get easily (omitting arguments):

$$
\begin{aligned}
& D_{u} L(u, \eta) v=-\int_{0}^{T}\left(\mathrm{~d} p_{u, \eta} z_{u, v}+p_{u, \eta} \dot{z}_{u, v} \mathrm{~d} t\right)+\phi_{y}\left(y_{u}(T)\right) z_{u, v}(T) \\
&+\int_{0}^{T} H_{u} v \mathrm{~d} t \\
& D_{u u}^{2} L(u, \eta)(v, v)= \int_{0}^{T} D^{2} H_{(u, y)^{2}}\left(v, z_{u, v}\right)^{2} \mathrm{~d} t+z_{u, v}(T)^{*} \phi_{y y}\left(y_{u}(T)\right) z_{u, v}(T) \\
&+\int_{0}^{T} z_{u, v}^{*} g_{y y}\left(y_{u}\right) z_{u, v} \mathrm{~d} \eta \\
&-\int_{0}^{T}\left(\mathrm{~d} p_{u, \eta} z_{u, v v}+p_{u, \eta} \dot{z}_{u, v v} \mathrm{~d} t\right)+\phi_{y}\left(y_{u}(T)\right) z_{u, v v}(T) .
\end{aligned}
$$

To obtain (9) and (10) it suffices, in view of Lemma $\mathbf{A . 2}$ to integrate by parts in the above expressions $p_{u, \eta}$ with $z_{u, v}$ and with $z_{u, v v}$, respectively.

First Order Necessary Condition. For $x \in K=C_{-}(0, T)$, define the first order contact set $I(x):=\{t \in[0, T] ; x(t)=0\}$. The expression of the tangent and normal cones (in the sense of convex analysis) to $K$ at point $x$, respectively $T_{K}(x)$ and $N_{K}(x)$, are well-known (see e.g. [4]) and given, for $x \in K$ (these sets being empty if $x \notin K$ ), by:

$$
\begin{aligned}
T_{K}(x) & =\{h \in C[0, T] ; h(t) \leq 0 \text { on } I(x)\}, \\
N_{K}(x) & =\left\{\eta \in \mathcal{M}_{+}[0, T] ; \operatorname{supp}(\eta) \subset I(x)\right\} .
\end{aligned}
$$

Here by $\operatorname{supp}(\eta)$ we denote the support of $\eta \in \mathcal{M}[0, T]$, i.e. the complement in $[0, T]$ of the largest open set $W \subset[0, T]$ that satisfies: $\int_{0}^{T} x(t) \mathrm{d} \eta(t)=0$, for all functions $x \in C[0, T]$ vanishing on $[0, T] \backslash W$. 
Let $u \in \mathcal{U}$. We say that $\eta \in \mathcal{M}_{+}[0, T]$ is a Lagrange multiplier associated with $u$ if the following first order necessary optimality condition holds:

$$
D_{u} L(u, \eta)=D J(u)+D G(u)^{*} \eta=0 \quad ; \quad \eta \in N_{K}(G(u)) .
$$

The set of Lagrange multipliers associated with $u$ is denoted by $\Lambda(u)$.

Robinson's constraint qualification (see [24, 25]) for problem (4) is as follows:

$$
\exists \varepsilon>0, \quad \varepsilon B_{C} \subset G(u)+D G(u) \mathcal{U}-K .
$$

Here $B_{C}$ denotes the unit (open) ball of $C[0, T]$.

The next theorem is well-known (see e.g. [4, Lemma 2.98 and Theorem 3.9). Note that for $v \in \mathcal{U}$, we have $D G(u) v=g_{y}\left(y_{u}\right) z_{u, v}$, i.e., $(D G(u) v)(t)=g_{y}\left(y_{u}(t)\right) z_{u, v}(t)$, for all $t \in[0, T]$.

Theorem 2.1. (i) A characterization of (14) is:

$$
\text { There exists } v \in \mathcal{U} ; \quad g_{y}\left(y_{u}(t)\right) z_{u, v}(t)<0 \text {, for all } t \in I\left(g\left(y_{u}\right)\right) \text {. }
$$

(ii) Let $u$ be a local solution of (4), satisfying (15). Then with $u$ is associated a non empty and bounded set of Lagrange multipliers.

Second Order Analysis. Let the critical cone be defined by:

$$
C(u)=\left\{v \in \mathcal{U} ; D G(u) v \in T_{K}(G(u)) ; D J(u) v \leq 0\right\} .
$$

For $h \in T_{K}(x)$, the second-order contact set is defined by:

$$
I^{2}(x, h)=\{t \in I(x) ; h(t)=0\} .
$$

If (13) holds, then $D J(u) v \geq 0$ for all $v$ such that $D G(u) v \in T_{K}(G(u))$ and $D J(u) v=0$ iff $\eta \perp D G(u) v$. Since $\eta \geq 0$ has support in $I(G(u))$, and $D G(u) v \leq 0$ on $I(G(u))$, we obtain the following (classical) statement:

Lemma 2.2. Let $(u, \eta)$ satisfy the first order necessary condition (13). Then:

$$
C(u)=\left\{v \in \mathcal{U} ; D G(u) v \in T_{K}(G(u)) ; \operatorname{supp}(\eta) \subset I^{2}(G(u), D G(u) v)\right\} .
$$

The inner and outer second-order tangent sets, respectively $T_{K}^{2, i}(x, h)$ and $T_{K}^{2}(x, h)$, are defined by:

$$
\begin{aligned}
T_{K}^{2, i}(x, h) & :=\left\{w \in C[0, T] ; \operatorname{dist}\left(x+\varepsilon h+\frac{1}{2} \varepsilon^{2} w, K\right)=o\left(\varepsilon^{2}\right), \varepsilon \geq 0\right\}, \\
T_{K}^{2}(x, h) & :=\left\{w \in C[0, T] ; \exists \varepsilon_{n} \downarrow 0, \operatorname{dist}\left(x+\varepsilon_{n} h+\frac{1}{2} \varepsilon_{n}^{2} w, K\right)=o\left(\varepsilon_{n}^{2}\right)\right\} .
\end{aligned}
$$

We recall the characterization of the inner second-order tangent set $T_{K}^{2, i}(x, h)$ due to Kawasaki [15, 14] (see also [7]): if $x \in K$ and $h \in T_{K}(x)$, then

$$
T_{K}^{2, i}(x, h)=\left\{w \in C[0, T] ; w(t) \leq \varsigma_{x, h}(t) \text { on }[0, T]\right\},
$$


where $\varsigma_{x, h}:[0, T] \rightarrow \overline{\mathbb{R}}$ is given by:

$$
\varsigma_{x, h}(t)= \begin{cases}0 & \text { if } t \in(\operatorname{int} I(x)) \cap I^{2}(x, h) \\ \liminf _{t^{\prime} \rightarrow t ; x(t)<0} \frac{\left(h(t)_{+}\right)^{2}}{2 x(t)} & \text { if } t \in \partial I(x) \cap I^{2}(x, h) \\ +\infty & \text { otherwise. }\end{cases}
$$

Here $h(t)_{+}:=\max \{h(t), 0\}$, and int $S$ and $\partial S$ denote respectively the interior and boundary of set $S$. Set $\mathcal{T}(x, h):=\partial I(x) \cap I^{2}(x, h)$. We have $\varsigma_{x, h}(\tau) \leq 0$ for $\tau \in \mathcal{T}(x, h)$ and it is not difficult to check that $t \mapsto \varsigma_{x, h}(t)$ is lower semi-continuous. Consequently, $T_{K}^{2, i}(x, h) \neq \emptyset$ iff $\varsigma_{x, h}(t)>-\infty$ for all $t$. In that case, $\varsigma_{x, h}$ is the upper limit of a increasing sequence of continuous functions $\left(\varsigma_{n}\right)$. Given $\eta \in \mathcal{M}_{+}[0, T]$, we may define (see e.g. [15]):

$$
\int_{0}^{T} \varsigma_{x, h}(t) \mathrm{d} \eta(t):=\sup \left\{\int_{0}^{T} \varsigma(t) \mathrm{d} \eta(t) ; \varsigma \leq \varsigma_{x, h}\right\} \in \mathbb{R} \cup\{+\infty\} .
$$

Then:

$$
\sigma\left(\eta, T_{K}^{2, i}(x, h)\right)=\int_{0}^{T} \varsigma_{x, h}(t) \mathrm{d} \eta(t),
$$

where $\sigma(\eta, S)=\sup _{w \in S}\langle\eta, w\rangle$ denotes the support function of the set $S$. If the support of $\eta$ satisfies $\operatorname{supp}(\eta) \subset I^{2}(x, h)$, then

$$
\sigma\left(\eta, T_{K}^{2, i}(x, h)\right) \leq 0
$$

A second-order necessary condition due to Kawasaki [13] is:

Theorem 2.2. Let $u$ be a local optimal solution of (4) satisfying (14). Then, for all $v \in$ $C(u)$, the following holds:

$$
\sup _{\eta \in \Lambda(u)}\left\{D_{u u}^{2} L(u, \eta)(v, v)-\sigma\left(\eta, T_{K}^{2, i}(G(u), D G(u) v)\right)\right\} \geq 0 .
$$

Remark 2.1. The above second-order necessary condition was improved by Cominetti in [6], by stating that for all convex set $\mathcal{S}_{u, v} \subset T_{K}^{2}(G(u), D G(u) v)$,

$$
\sup _{\eta \in \Lambda(u)}\left\{D_{u u}^{2} L(u, \eta)(v, v)-\sigma\left(\eta, \mathcal{S}_{u, v}\right)\right\} \geq 0
$$

Th. 2.2 is obtained for the particular choice of $\mathcal{S}_{u, v}=T_{K}^{2, i}(G(u), D G(u) v)$. For the problem considered in the present paper, we gain sufficient information from (23) (see Proposition 3.1. 


\subsection{Junction Condition Analysis}

We first recall some classical definitions. A boundary (resp. interior) arc is a maximal interval of positive measure $\mathcal{I} \subset[0, T]$ such that $g(y(t))=0$ (resp. $g(y(t))<0)$ for all $t \in \mathcal{I}$. If $\left[\tau_{e n}, \tau_{e x}\right]$ is a boundary arc, $\tau_{e n}$ and $\tau_{e x}$ are called entry and exit point, respectively. Entry and exit points are said to be regular if they are endpoint of an interior arc. A touch point $\tau$ in $(0, T)$ is an isolated contact point (endpoint of two interior arcs). Entry, exit and touch points are called junctions points (or times). We say that the junctions are regular, when the junctions points are regular. In this paper, only the case of finitely many regular junctions is dealt with.

The first-order time derivative of the state constraint when $y$ satisfies the state equation (2), i.e., $g^{(1)}(u, y)=\frac{\mathrm{d}}{\mathrm{d} t} g(y(t))=g_{y}(y) f(u, y)$, is denoted by $g^{(1)}(y)$ if the function $\mathbb{R} \times \mathbb{R}^{n} \rightarrow$ $\mathbb{R} ;(u, y) \mapsto g_{y}(y) f(u, y)$ does not depend on $u$ (that is, the function $(u, y) \mapsto g_{u}^{(1)}(u, y)$ is identically zero). We may define similarly $g^{(2)}, \ldots, g^{(q)}$ if $g, f$ are $C^{q}$ and if $g_{u}^{(j)} \equiv 0$, for all $j=1, \ldots, q-1$, and we have $g^{(j)}(u, y)=g_{y}^{(j-1)}(y) f(u, y)$, for $j=1, \ldots, q$.

Let $q \geq 1$ be the smallest number of times derivations of the state constraint, so that a dependence w.r.t. $u$ appears. If $q$ is finite, we say that $q$ is the order of the state constraint (see e.g. [5]).

Let $u \in \mathcal{U}$ be a solution of the first order necessary condition (13), with Lagrange multiplier $\eta$ and costate $p_{u, \eta}$ solution of (7). Since $\eta$ and $p_{u, \eta}$ are of bounded variation, they have at most countably many discontinuity times, and are everywhere on $[0, T]$ left and right continuous. We denote by $[\eta(\tau)]=\eta\left(\tau^{+}\right)-\eta\left(\tau^{-}\right)$where $\eta\left(\tau^{ \pm}\right)=\lim _{t \rightarrow \tau^{ \pm}} \eta(t)$ the jump discontinuity of $\eta$ at time $\tau \in[0, T]$. We make the following assumptions:

(A2) The Hamiltonian is strongly convex w.r.t. the control variable, uniformly w.r.t. $t \in$ $[0, T]$ :

$$
\exists \gamma>0, \quad H_{u u}\left(\hat{u}, y_{u}(t), p_{u, \eta}\left(t^{ \pm}\right)\right) \geq \gamma \quad \forall \hat{u} \in \mathbb{R}, \forall t \in[0, T] .
$$

(A3) (Constraint regularity) The data of the problem are $C^{2 q}$, i.e. $k \geq 2 q$ in (A0), the state constraint is of order $q$ and the condition below holds:

$$
\exists \beta>0, \quad\left|g_{u}^{(q)}\left(\hat{u}, y_{u}(t)\right)\right|>\beta, \quad \forall \hat{u} \in \mathbb{R}, \forall t \in[0, T] .
$$

(A4) The trajectory $\left(u, y_{u}\right)$ has a finite set of junctions times, that will be denoted by $\mathcal{T}=: \mathcal{T}_{\text {en }} \cup \mathcal{T}_{\text {ex }} \cup \mathcal{T}_{\text {to }}$, with $\mathcal{T}_{\text {en }}, \mathcal{T}_{\text {ex }}$ and $\mathcal{T}_{\text {to }}$ the disjoint (and possibly empty) subsets of respectively regular entry, exit and touch points, and we suppose that $g\left(y_{u}(T)\right)<0$.

Remark 2.2. An assumption weaker than (A2), that is enough for the sufficient conditions in section 4 and 5 is

(A2') (strengthened Legendre-Clebsch condition)

$$
\exists \gamma>0, \quad H_{u u}\left(u(t), y_{u}(t), p_{u, \eta}(t)\right) \geq \gamma \quad \text { a.e. } t \in[0, T] .
$$


F. Bonnans \& A. Hermant

A touch point $\tau \in \mathcal{T}_{\text {to }}$ is said to be essential, if the Lagrange multiplier $\eta$ satisfies $[\eta(\tau)]>0$. The set of essential touch points of the trajectory $\left(u, y_{u}\right)$ will be denoted by $\mathcal{T}_{\text {to }}^{\text {ess }}$.

The above hypotheses imply the continuity of the control variable and of some of its derivatives at junction points. The next proposition is due to Jacobson et al. [12. Its proof was later clarified in Maurer [19].

Proposition 2.1. Let $u \in \mathcal{U}$ satisfying (13) with Lagrange multiplier $\eta$ and assume that (A2)-(A4) hold. Then:

(i) The control $u$ is continuous over $[0, T]$ (in particular at junctions points $\tau \in \mathcal{T}$ ) and $C^{q}$ on $[0, T] \backslash \mathcal{T}$. The multiplier $\eta$ is continuously differentiable on $[0, T] \backslash \mathcal{T}$.

(ii) If $\tau \in \mathcal{T}_{\text {en }} \cup \mathcal{T}_{\text {ex }}$ is a regular entry or exit point, then: (a) if $q$ is odd, $\eta$ and the $q-1$ first time derivatives of $u$ are continuous at $\tau$; (b) if $q$ is even, the $q-2$ first time derivatives of $u$ are continuous at $\tau$.

(iii) If $\tau \in \mathcal{T}_{\text {to }}$ is a touch point, then: (a) the $q-2$ first derivatives of $u$ are continuous at $\tau$; (b) if $q=1$, then $\eta$ and $\dot{u}$ are also continuous at $\tau$ (that is, if $q=1$, then $\left(u, y_{u}\right)$ does not have essential touch point).

Remark 2.3. Under the assumptions of Prop. 2.1] we have the following decomposition: $\mathrm{d} \eta(t)=\eta_{0}(t) \mathrm{d} t+\sum_{\tau \in \mathcal{T}} \nu_{\tau} \delta_{\tau}(t)$ where $\delta_{\tau}$ denotes the Dirac measure at time $\tau$, the density $\eta_{0} \in L^{1}(0, T)$ is equal to $\frac{\mathrm{d} \eta}{\mathrm{d} t}$ almost everywhere and $\nu_{\tau}:=[\eta(\tau)] \geq 0$. We have $\nu_{\tau}=0$ if $q$ is odd and $\tau$ is a regular entry/exit point, and if $q=1$ and $\tau$ is a touch point.

We end this section by a result on constraint qualification and uniqueness of the multiplier. For this we need the expression of the time derivatives of $D G(u) v$.

Lemma 2.3. Assume that $f, g$ are $C^{q}$ and that $g_{u}^{(j)} \equiv 0$, for $j=1, \ldots, q-1$. Then: (i) For all $v \in \mathcal{U}$, the following relations hold:

$$
\begin{aligned}
& \frac{\mathrm{d}^{j}}{\mathrm{~d} t^{j}} g_{y}\left(y_{u}\right) z_{u, v}=g_{y}^{(j)}\left(u, y_{u}\right) z_{u, v}, \quad j=1, \ldots, q-1, \\
& \frac{\mathrm{d}^{q}}{\mathrm{~d} t^{q}} g_{y}\left(y_{u}\right) z_{u, v}=g_{y}^{(q)}\left(u, y_{u}\right) z_{u, v}+g_{u}^{(q)}\left(u, y_{u}\right) v .
\end{aligned}
$$

(ii) If in addition, (26) is satisfied, then $D G(u)$ is an isomorphism between $L^{\infty}(0, T)$ and the space $\mathcal{W}$ defined by:

$$
\mathcal{W}:=\left\{\varphi \in W^{q, \infty}(0, T) ; \varphi^{(j)}(0)=0 ; j=0, \ldots, q-1\right\} .
$$

Proof. (i) By (8), we have:

$$
\begin{aligned}
\frac{\mathrm{d}}{\mathrm{d} t} g_{y}\left(y_{u}\right) z_{u, v} & =g_{y y}\left(y_{u}\right) f\left(u, y_{u}\right) z_{u, v}+g_{y}\left(y_{u}\right) f_{y}\left(u, y_{u}\right) z_{u, v}+g_{y}\left(y_{u}\right) f_{u}\left(u, y_{u}\right) v \\
& =g_{y}^{(1)}\left(u, y_{u}\right) z_{u, v}+g_{u}^{(1)}\left(u, y_{u}\right) v .
\end{aligned}
$$


Since $g_{u}^{(j)} \equiv 0$ for $j=1$ to $q-1$, we obtain by induction that $\frac{\mathrm{d}^{j}}{\mathrm{~d} t^{j}} g_{y}\left(y_{u}\right) z_{u, v}=g_{y}^{(j)}\left(u, y_{u}\right) z_{u, v}$ is independent on $v$, and that the derivative of order $q$ has the expression in (29).

(ii) If in addition (26) is satisfied, it is easily seen by (29) that for all $\varphi \in \mathcal{W}$, there exists a unique $v \in \mathcal{U}$ such that $g_{y}\left(y_{u}\right) z_{u, v}=\varphi$. The conclusion follows from the open mapping theorem.

Proposition 2.2. Assume that (A1) holds, and let $u \in \mathcal{U}$ satisfy (A3). Then: (i) Robinson's constraint qualification [14) holds; (ii) if $\Lambda(u) \neq \emptyset$, the Lagrange multiplier $\eta$ associated with $u$ is unique.

Proof. It is obvious by Lemma 2.3(ii) and Lemma 2.1(i) that (14) holds iff (A1) does. This proves (i). Assume that $\eta_{1}, \eta_{2} \in \Lambda(u)$ and set $\mu:=\eta_{2}-\eta_{1} \in \mathcal{M}[0, T]$. Since $D G(u)^{*} \mu=0$, it follows that $\int_{0}^{T} \varphi(t) \mathrm{d} \mu(t)=0$, for all $\varphi \in \mathcal{W}$. Since $g\left(y_{0}\right)<0$, we have $\operatorname{supp}(\mu) \subset[2 \varepsilon, T]$ for some $\varepsilon>0$. Taking the restriction to $[\varepsilon, T]$ of functions in $D G(u) \mathcal{U}$, we obtain the whole space $W^{q, \infty}(\varepsilon, T)$. By density of the latter in $C[\varepsilon, T]$ we deduce that for all $\varphi \in C[0, T]$, $\int_{0}^{T} \varphi(t) \mathrm{d} \mu(t)=\int_{\varepsilon}^{T} \varphi(t) \mathrm{d} \mu(t)=0$. Hence $\mathrm{d} \mu \equiv 0$, which achieves the proof of (ii).

\section{Second-order Necessary Conditions}

\subsection{Basic Second-order Necessary Conditions}

Let $u \in \mathcal{U}$ satisfy assumptions (A2)-(A4) and $\eta \in \Lambda(u)$. We make the following assumptions. Let $\hat{q}:=2 q-1$ if $q$ is odd and $\hat{q}:=2 q-2$ if $q$ is even.

(A5) (Non Tangentiality Condition)

(i) For all entry time $\tau_{e n} \in \mathcal{T}_{\text {en }}$ and all exit time $\tau_{e x} \in \mathcal{T}_{\text {ex }}$ :

$$
\left.(-1)^{\hat{q}+1} \frac{\mathrm{d}^{\hat{q}+1}}{\mathrm{~d} t^{\hat{q}+1}} g\left(y_{u}(t)\right)\right|_{t=\tau_{e n}^{-}}<0 ;\left.\quad \frac{\mathrm{d}^{\hat{q}+1}}{\mathrm{~d} t^{\hat{q}+1}} g\left(y_{u}(t)\right)\right|_{t=\tau_{e x}^{+}}<0 .
$$

(ii) For all essential touch point $\tau_{t o} \in \mathcal{T}_{\text {to }}^{\text {ess }}$ :

$$
\left.\frac{\mathrm{d}^{2}}{\mathrm{~d} t^{2}} g\left(y_{u}(t)\right)\right|_{t=\tau_{t o}}<0 .
$$

(A6) (Strict Complementarity on boundary arcs): int $I(G(u)) \subset \operatorname{supp}(\eta)$.

Remark 3.1. 1) By Proposition 2.1, the expressions appearing in assumption (A5)(i)(ii) are well-defined, and $\hat{q}+1$ is the smallest possible order for which the corresponding derivative of $g\left(y_{u}\right)$ may be nonzero at an entry or exit point. Note that $\hat{q}=q$ for $q=1,2$.

2) Only the assumption (A6') below, weaker than (A6), is used in necessary condition of Theorem 3.1 in order to ensure that the second-order tangent set $T_{K}^{2, i}(G(u), D G(u) v)$ is not empty, for all $v \in C(u)$ : 
(A6') (Strict Complementarity near entry/exit of boundary arcs): For all entry point $\tau_{e n} \in$ $\mathcal{T}_{\text {en }}$ and exit point $\tau_{e x} \in \mathcal{T}_{\text {ex }}$, there exists $\varepsilon>0$ such that:

$$
\left(\tau_{e n}, \tau_{e n}+\varepsilon\right) \subset \operatorname{supp}(\eta) ;\left(\tau_{e x}-\varepsilon, \tau_{e x}\right) \subset \operatorname{supp}(\eta) .
$$

Note that we do not assume strict complementarity at touch points.

Theorem 3.1. Assume that (A1) holds. Let $u \in \mathcal{U}$ be an optimal solution of (4), with its Lagrange multiplier $\eta$, satisfying (A2)-(A5) and (A6)'. Let $\mathcal{T}_{\text {to }}^{\text {ess }}$ denote the (finite) set of essential touch points of the trajectory $\left(u, y_{u}\right)$ and $\nu_{\tau}=[\eta(\tau)]>0$, for $\tau \in \mathcal{T}_{\text {to }}^{\text {ess }}$. Then, for all $v \in C(u)$ :

$$
D_{u u}^{2} L(u, \eta)(v, v)-\sum_{\tau \in \mathcal{T}_{t o}^{\text {ess }}} \nu_{\tau} \frac{\left(g_{y}^{(1)}\left(y_{u}(\tau)\right) z_{u, v}(\tau)\right)^{2}}{\left.\frac{\mathrm{d}^{2}}{\mathrm{~d} t^{2}} g\left(y_{u}(t)\right)\right|_{t=\tau}} \geq 0 .
$$

Corollary 3.1. Under the assumptions of Theorem 3.1, if the trajectory $\left(u, y_{u}\right)$ has no essential touch point (in particular, if the state constraint is of first order $q=1$ ), then $D_{u u}^{2} L(u, \eta)(v, v) \geq 0$, for all $v \in C(u)$.

In the sequel, we denote $I^{2}(G(u), D G(u) v)$ by $I_{u, v}^{2}$. For all $v \in C(u)$, by (18), we have $\mathcal{T}_{\text {to }}^{\text {ess }} \subset\left(\mathcal{T}_{\text {to }} \cap I_{u, v}^{2}\right)$. Let us denote the subset of critical directions that "avoid" non essential touch point (i.e., such that $g\left(y_{u}(\tau)\right) z_{u, v}(\tau)<0$, for all $\tau \in \mathcal{T}_{\text {to }} \backslash \mathcal{T}_{\text {to }}^{\text {ess }}$ ) by:

$$
C_{0}(u):=\left\{v \in C(u) ; \mathcal{T}_{\text {to }} \cap I_{u, v}^{2}=\mathcal{T}_{\text {to }}^{\text {ess }}\right\} .
$$

The first step of the proof of Theorem 3.1 consists in computing the sigma-term for the critical directions in $C_{0}(u)$.

Proposition 3.1. Let $v \in C_{0}(u)$. Under the assumptions of Theorem [3.1, we have that

$$
\sigma\left(\eta, T_{K}^{2, i}(G(u), D G(u) v)\right)=\sum_{\tau \in \mathcal{T}_{\text {tos }}^{\text {ess }}} \nu_{\tau} \frac{\left(g_{y}^{(1)}\left(y_{u}(\tau)\right) z_{u, v}(\tau)\right)^{2}}{\left.\frac{\mathrm{d}^{2}}{\mathrm{~d} t^{2}} g\left(y_{u}(t)\right)\right|_{t=\tau}} .
$$

Proof. The proof is divided into 3 steps. We first analyse the contribution of entry/exit points, then the one of touch points, and finally conclude.

Remind that by (20), only the points in $\partial I(G(u)) \cap I_{u, v}^{2}$ have a contribution to the sigma term. Note that $\partial I(G(u))=\mathcal{T}$. Set $\varsigma_{u, v}:=\varsigma_{g\left(y_{u}\right), g_{y}\left(y_{u}\right) z_{u, v}}=\varsigma_{G(u), D G(u) v}$ and let $\tau \in \mathcal{T} \cap I_{u, v}^{2}$. By (20), we have:

$$
\varsigma_{u, v}(\tau)=\liminf _{t \rightarrow \tau ; g\left(y_{u}(t)\right)<0} \frac{\left(\left\{g_{y}\left(y_{u}\right) z_{u, v}(t)\right\}_{+}\right)^{2}}{2 g\left(y_{u}(t)\right)} .
$$

1) (Entry/exit point). Assume that $\tau \in \mathcal{T}_{\text {en }} \cup \mathcal{T}_{\text {ex }}$. According to Prop. 2.1(ii), time derivatives of the control at regular entry/exit points are continuous until order $q-2$ if $q$ is even, and $q-1$ if $q$ is odd. Consequently, by definition of the order of the state constraint, 
the times derivatives of $g\left(y_{u}\right)$ are continuous at $\tau$ until order $2 q-2$ is $q$ is even, and $2 q-1$ if $q$ is odd. Hence they all vanish at entry/exit time $\tau$ of a boundary arc. It follows that for $t$ at neighborhood of $\tau$ on the interior arc side, a Taylor expansion gives, by definition of $\hat{q}$ :

$$
g\left(y_{u}(t)\right)=\left.\frac{\mathrm{d}^{\hat{q}+1}}{\mathrm{~d} t^{\hat{q}+1}} g\left(y_{u}\right)\right|_{t=\tau^{ \pm}} \frac{(t-\tau)^{\hat{q}+1}}{(\hat{q}+1) !}+o\left((t-\tau)^{\hat{q}+1}\right),
$$

where, for the sake of simplicity, we denote by $\tau^{ \pm}$either $\tau^{-}$if $\tau \in \mathcal{T}_{\text {en }}$ or $\tau^{+}$if $\tau \in \mathcal{T}_{\text {ex }}$.

Combining Lemma 2.2 and (A6'), we see that for all $v \in C(u)$, the function (of time) $g_{y}\left(y_{u}\right) z_{u, v}$ vanishes just after entering or before leaving a boundary arc on a small interval $[\tau, \tau \pm \varepsilon]$, and so do its first $q-1$ time derivatives since the latter are continuous by Lemma 2.3 (i). The $q$-th derivative of $g_{y}\left(y_{u}\right) z_{u, v}$ being a bounded function by (29), we have, on the interior arc side:

$$
\left|g_{y}\left(y_{u}(t)\right) z_{u, v}(t)\right| \leq C|t-\tau|^{q} .
$$

If $q$ is odd, combining (37) with $\hat{q}=2 q-1$ and (38) and by tangentiality assumption (A5)(i), we deduce from (36) that:

$$
\varsigma_{u, v}(\tau) \geq \lim _{t \rightarrow \tau^{ \pm}} \frac{C^{2}(t-\tau)^{2 q}}{\left.\frac{\mathrm{d}^{2 q}}{\mathrm{~d} t^{2 q}} g\left(y_{u}\right)\right|_{t=\tau^{ \pm}} \frac{(t-\tau)^{2 q}}{(2 q) !}+o\left((t-\tau)^{2 q}\right)}>-\infty .
$$

If $q$ is even, (37) with $\hat{q}=2 q-2$, 38) and (A5) (i) in (36) give:

$$
\varsigma_{u, v}(\tau) \geq \lim _{t \rightarrow \tau^{ \pm}} \frac{C^{2}(t-\tau)^{2 q}}{\left.\frac{\mathrm{d}^{2 q-1}}{\mathrm{~d}^{2 q-1}} g\left(y_{u}\right)\right|_{t=\tau^{ \pm}} \frac{(t-\tau)^{2 q-1}}{(2 q-1) !}+o\left((t-\tau)^{2 q-1}\right)}=0 .
$$

Since $\varsigma_{u, v}(\tau) \leq 0$ by (20) at an entry or exit point, it follows that (when $q$ is even) $\varsigma_{u, v}(\tau)=0$.

2) (Touch point). Assume now that $\tau \in \mathcal{T}_{t o} \cap I_{u, v}^{2}$. If that case happens, since $v \in C_{0}(u)$, our hypotheses imply that $\tau$ is an essential touch point satisfying (32), and hence, that $q \geq 2$. Since $g\left(y_{u}\right)$ has a isolated local maximum at $\tau, g\left(y_{u}\right)$ and $g^{(1)}\left(y_{u}\right)$ vanish at $\tau$ while $\frac{\mathrm{d}}{\mathrm{d} t} g^{(1)}=g^{(2)}\left(u, y_{u}\right)$ is nonpositive and continuous at $\tau$ since $u$ is continuous by Prop. 2.1 (i). We thus have:

$$
g\left(y_{u}(t)\right)=\left.\frac{\mathrm{d}}{\mathrm{d} t} g^{(1)}\left(y_{u}\right)\right|_{t=\tau} \frac{(t-\tau)^{2}}{2}+o\left((t-\tau)^{2}\right) .
$$

Since $\tau \in I_{u, v}^{2}$, we also have $g_{y}\left(y_{u}(\tau)\right) z_{u, v}(\tau)=0$. The function $g_{y}\left(y_{u}\right) z_{u, v}$ being $C^{1}$ (since $q \geq 2$ ) with almost everywhere a bounded second derivative, we get by [28), taking the nonnegative part:

$$
\left(g_{y}\left(y_{u}(t)\right) z_{u, v}(t)\right)_{+}=\left(g_{y}^{(1)}\left(y_{u}(\tau)\right) z_{u, v}(\tau)(t-\tau)\right)_{+}+o(t-\tau) .
$$

From (39), (40) and (A5)(ii), $\left(g_{u}\left(y_{u}\right) z_{u, v}\right)_{+}^{2} / g\left(y_{u}\right)$ is left-and right continuous when $t \rightarrow \tau$. Therefore, taking the lim inf when $t \rightarrow \tau$ comes to take the min of both limits when $t \rightarrow \tau^{+}$ and $t \rightarrow \tau^{-}$, thus we obtain:

$$
\varsigma_{u, v}(\tau)=\min \left\{\frac{\left(g_{y}^{(1)}\left(y_{u}(\tau)\right) z_{u, v}(\tau)\right)^{2}}{g^{(2)}\left(u(\tau), y_{u}(\tau)\right)} ; 0\right\}=\frac{\left(g_{y}^{(1)}(\bar{y}(\tau)) z_{\bar{u}, v}(\tau)\right)^{2}}{g^{(2)}\left(u(\tau), y_{u}(\tau)\right)}>-\infty .
$$


3) (Conclusion). For all $\tau \in \mathcal{T} \cap I_{u, v}^{2}$, we showed that $\varsigma_{u, v}(\tau)>-\infty$. Therefore we may apply (21). Set $I_{0}:=\operatorname{int} I(G(u))$. By (18), we have $\operatorname{supp}(\eta) \subset I_{u, v}^{2}$ and in view of remark 2.3 we may write that:

$$
\sigma\left(\eta, T_{K}^{2, i}(G(u), D G(u) v)=\int_{I_{0}} \varsigma_{u, v}(t) \eta_{0}(t) \mathrm{d} t+\sum_{\tau \in \mathcal{T} \cap I_{u, v}^{2}} \nu_{\tau} \varsigma_{u, v}(\tau)\right.
$$

where $\eta_{0} \in L^{1}\left(I_{0}\right)$ and $\nu_{\tau}=[\eta(\tau)]$. By (20), $\varsigma_{u, v}$ vanishes on $I_{0} \cap I_{u, v}^{2}$ and thus on $I_{0} \cap$ $\operatorname{supp}\left(\eta_{0}\right)$. Hence, $\int_{I_{0}} \varsigma_{u, v}(t) \eta_{0}(t) \mathrm{d} t=0$. If $\tau \in \mathcal{T}_{\text {en }} \cup \mathcal{T}_{\text {ex }}$, we have, if $q$ is odd, $\nu_{\tau}=0$ by Prop. 2.11ii) (a) and we showed that $\varsigma_{u, v}(\tau)>-\infty$. If $q$ is even, we showed in point 1) that $\varsigma_{u, v}(\tau)=0$ (and we have $\nu_{\tau}<+\infty$ ). In both cases, we deduce that $\nu_{\tau} \varsigma_{u, v}(\tau)=0$.

It remains only in (42), when $q \geq 2$, the contribution of finitely many touch points $\tau$ in $\mathcal{T}_{t o} \cap I_{u, v}^{2}=\mathcal{T}_{t o}^{\text {ess }}$ with $\varsigma_{u, v}(\tau)$ given by (41). Hence (35) follows.

Proof of Theorem 3.1. Combining Theorem 2.2 and Propositions 2.2] and 3.1, we obtain that (34) holds, for all $v \in C_{0}(u)$. Since the left-hand-side of (34) is a continuous quadratic form, it remains nonnegative on the closure of $C_{0}(u)$. We end the proof by checking that the latter is equal to $C(u)$, the cone of critical directions.

Since $C(u)$ is closed and contains $C_{0}(u)$, we have of course $\overline{C_{0}(u)} \subset C(u)$. We prove the converse relation. Let $v_{0} \in C(u)$. We remind that $v \in C(u)$ iff $g_{y}\left(y_{u}\right) z_{u, v} \leq 0$ on $I\left(g\left(y_{u}\right)\right)$ and $g_{y}\left(y_{u}\right) z_{u, v}=0$ on the support of the Lagrange multiplier $\eta$. Let $\rho: \mathbb{R} \rightarrow \mathbb{R}$ be a function of class $C^{\infty}$ having support on $[-1,1]$, and positive on $(-1,1)$. For $\varepsilon>0$, set $\rho_{\varepsilon}(t):=\varepsilon^{q+1} \rho(t / \varepsilon)$, thus we have $\rho_{\varepsilon} \rightarrow 0$ in $W^{q, \infty}$. By Lemma 2.3 (ii), for $\varepsilon>0$ small enough, there exists a unique $v_{\varepsilon} \in L^{\infty}(0, T)$ such that $g\left(y_{u}\right) z_{u, v_{\varepsilon}}=g\left(y_{u}\right) z_{u, v_{0}}-\sum_{t \in \mathcal{T}_{t o} \backslash \mathcal{T}_{\text {to }}^{\text {ess }}} \rho_{\varepsilon}(t-$ $\tau) \in W^{q, \infty}(0, T)$. Then we have $g_{y}\left(y_{u}\right) z_{u, v_{\varepsilon}}=g_{y}\left(y_{u}\right) z_{u, v_{0}}$ outside $(\tau-\varepsilon, \tau+\varepsilon)$, for all non essential touch point $\tau, g_{y}\left(y_{u}(\tau)\right) z_{u, v_{\varepsilon}}(\tau)<0$ for such $\tau$, and hence, the touch points being isolated, for $\varepsilon>0$ small enough, $v_{\varepsilon} \in C_{0}(u)$. Since $D G(u) v_{\varepsilon} \rightarrow D G(u) v_{0}$ in $\mathcal{W}$, where $\mathcal{W}$ was defined in (30), and $D G(u)$ has a bounded inverse by Lemma [2.3)(ii), we have $v_{\varepsilon} \rightarrow v_{0}$ in $L^{\infty}(0, T)$ when $\varepsilon \downarrow 0$. The conclusion follows.

\subsection{Extended Second-order Necessary Conditions}

The solution $z_{u, v}$ of the linearized state equation when $v \in L^{2}(0, T)$, is well-defined and belongs to $H^{1}(0, T) \subset C[0, T]$. Thus we may extend continuously $D J(u)$ and $D G(u)$ over $L^{2}(0, T)$ (we keep the same notations for the extensions). Since $D G(u): L^{2}(0, T) \rightarrow C[0, T]$, it makes sense to the extend the critical cone $C(u)$ to critical directions in $L^{2}$, as follows:

$$
C_{L^{2}}(u)=\left\{v \in L^{2}(0, T) \backslash D G(u) v \in T_{K}(G(u)) ; D J(u) v=0\right\} .
$$

The necessary and sufficient second-order conditions involve respectively $C(u)$ and $C_{L^{2}}(u)$ (see sections 4 and 5 ). Therefore, to obtain the no-gap second-order conditions, we need the following variant of Theorem 3.1 
Corollary 3.2. The statements of Theorem [3.1 and Corollary 3.1 still hold replacing assumption $\left(A 6^{\prime}\right)$ by $(A 6)$ and $C(u)$ by $C_{L^{2}}(u)$.

Corollary 3.2 is obtained as a consequence of Th. 3.1, continuity of the left-hand side of (34) w.r.t. $v \in L^{2}$, and density of $C(u)$ into $C_{L^{2}}(u)$ (Lemma 3.2). To prove the latter, we first need a general result.

Lemma 3.1. Let $q \geq 1$ and $a<b \in \mathbb{R}$. Then for all $\hat{x} \in H^{q}(a, b)=W^{q, 2}(a, b)$, there exists a sequence $\left(x_{n}\right)$ of $W^{q, \infty}(a, b)$ such that $x_{n}^{(j)}(a)=\hat{x}^{(j)}(a), x_{n}^{(j)}(b)=\hat{x}^{(j)}(b)$ for all $j=0, \ldots, q-1, n \in \mathbb{N}$ and $\left\|x_{n}-\hat{x}\right\|_{q, 2} \rightarrow 0$.

Proof. Set $\hat{x}_{a}:=\left(\hat{x}(a), \ldots, \hat{x}^{(q-1)}(a)\right)^{*}, \hat{x}_{b}:=\left(\hat{x}(b), \ldots, \hat{x}^{(q-1)}(b)\right)^{*} \in \mathbb{R}^{q}$ and $\hat{u}:=\hat{x}^{(q)} \in$ $L^{2}(a, b)$. For $u \in L^{2}(a, b)$, let $x_{u} \in H^{q}(a, b)$ be the solution of:

$$
x_{u}^{(q)}(t)=u(t) \quad \text { a.e. on }[a, b] \quad ; \quad\left(x_{u}(a), \ldots, x_{u}^{(q-1)}(a)\right)=\hat{x}_{a}^{*} .
$$

For $n \in \mathbb{N}$, consider the following problem:

$$
\left(\mathcal{P}_{n}\right) \quad \min \frac{1}{2}\|u-\hat{u}\|_{2}^{2} \quad ; \quad \mathcal{A} u=\hat{x}_{b} \quad ; \quad u \in \mathcal{U}_{n},
$$

where $\mathcal{U}_{n}:=\left\{u \in L^{2}(0, T) ;|u(t)| \leq n\right.$ a.e. $\}$ and $\mathcal{A}: L^{2} \rightarrow \mathbb{R}^{q} ; u \mapsto\left(x_{u}(b), \ldots, x_{u}^{(q-1)}(b)\right)^{*}$. By construction, $\mathcal{A} \hat{u}=\hat{x}_{b}$. It is readily seen that the mapping $L^{2}(a, b) \rightarrow H^{q}(a, b) ; u \mapsto x_{u}$ solution of (44) is continuous. Since $H^{q}(a, b)$ has a continuous inclusion into $C^{q-1}[a, b]$, it follows that the linear mapping $\mathcal{A}$ is also continuous.

Let us first show that for $n$ large enough, the problems $\left(\mathcal{P}_{n}\right)$ are feasible and uniformly qualified, that is there exist $n_{0} \in \mathbb{N}$ and $\delta_{0}>0$ such that

$$
\hat{x}_{b}+\delta_{0} B_{\mathbb{R}^{q}} \subset \mathcal{A} \mathcal{U}_{n_{0}} \subset \mathcal{A} \mathcal{U}_{n} \quad \forall n \geq n_{0},
$$

with $B_{\mathbb{R}^{q}}$ the unit ball in $\mathbb{R}^{q}$. Indeed, consider e.g. for $\delta \in \mathbb{R}^{q}$ the (unique) polynomial function $x_{\delta}$ on of degree $2 q-1$ that takes with its $q-1$ first derivatives the values $\hat{x}_{a}$ and $\hat{x}_{b}$ at $a$ and $b$. It is easily seen that its coefficients are solution of a full-rank linear system with $\hat{x}_{b}-\hat{x}_{a}+\delta$ as right-hand side, hence, taking the sup over $(t, \delta) \in[a, b] \times B_{\mathbb{R}^{q}}\left(0, \delta_{0}\right)$ of the functions $u_{\delta}(t)=x_{\delta}^{(q)}(t)$ that are $C^{\infty}$ w.r.t. $t$ and $\delta$ provides an uniform bound $n_{0}$ such that (46) holds.

Since Robinson's constraint qualification holds for $n$ large enough, there exists a (unique) optimal solution $u_{n}$ of $\left(\mathcal{P}_{n}\right)$ and a normal Lagrange multiplier $\lambda_{n} \in \mathbb{R}^{q *}$, such that (throughout the proof, $\langle\cdot, \cdot\rangle$ denotes the scalar product over $\left.L^{2}\right)$ :

$$
0 \leq\left\langle u_{n}-\hat{u}+\mathcal{A}^{*} \lambda_{n}, v-u_{n}\right\rangle \quad \forall v \in \mathcal{U}_{n} .
$$

Since the feasible set of problem $\left(\mathcal{P}_{n}\right)$ is increasing for inclusion when $n \rightarrow+\infty$, the cost function is decreasing, thus $\left\|u_{n}-\hat{u}\right\|_{2}$ is bounded. Hence the sequence $\left(u_{n}\right)$ converges weakly to some $\bar{u} \in L^{2}$. We may rewrite (47) as:

$$
\left\|u_{n}-\hat{u}\right\|_{2}^{2}+\lambda_{n}\left(\hat{x}_{b}-\mathcal{A} v\right) \leq\left\langle u_{n}-\hat{u}, v-\hat{u}\right\rangle \quad \forall v \in \mathcal{U}_{n} .
$$


Qualification property (46) implies that $\delta_{0}\left|\lambda_{n}\right| \leq \sup _{v \in \mathcal{U}_{n_{0}}} \lambda_{n}\left(x_{b}-\mathcal{A} v\right)$, hence, taking the sup for $v \in \mathcal{U}_{n_{0}}$ successively in the right and left hand side of (48), we deduce that for some constant $K\left(n_{0}\right)>0$ that depends on $n_{0}$, we have $\delta_{0}\left|\lambda_{n}\right| \leq K\left(n_{0}\right)$, for all $n \geq n_{0}$. Therefore the sequence $\left(\lambda_{n}\right)$ is uniformly bounded. Define now $v_{n} \in \mathcal{U}_{n}$ as $v_{n}(t)=\max \{-n ; \min \{n, \hat{u}(t)\}\}$ a.e. By the Lebesgue dominated convergence Theorem, $v_{n} \rightarrow \hat{u}$ in $L^{2}$ and by (48):

$$
\left\|u_{n}-\hat{u}\right\|_{2}^{2} \leq\left\langle u_{n}-\hat{u}, v_{n}-\hat{u}\right\rangle+\lambda_{n}\left(\mathcal{A} v_{n}-\hat{x}_{b}\right) \longrightarrow 0
$$

since $u_{n}-\hat{u} \rightarrow \bar{u}-\hat{u}$ weakly in $L^{2}, v_{n}-\hat{u} \rightarrow 0$ strongly in $L^{2}, \lambda_{n}$ is bounded and $\mathcal{A} v_{n} \rightarrow \mathcal{A} \hat{u}=\hat{x}_{b}$ by continuity of $\mathcal{A}$. It follows that $\left\|u_{n}-\hat{u}\right\|_{2} \rightarrow 0$ and the sequence $x_{n}:=x_{u_{n}}$ satisfies all the required properties, so the proof is completed.

Lemma 3.2. Let $u \in \mathcal{U}$ and $\eta \in \Lambda(u)$ such that (A3), (A4) and (A6) are satisfied. Then $C_{L^{2}}(u)$ is dense into $C(u)$.

Proof. Since (A4) holds, denote by $0<\tau_{1}<\ldots<\tau_{N}<T$ the junctions times of the trajectory $\left(u, y_{u}\right)$, and set $\tau_{0}:=0, \tau_{N+1}:=T$. Let $v \in C_{L^{2}}(u)$ and set $x:=D G(u) v$. By Lemma 3.1] applied on intervals $\left[\tau_{k}, \tau_{k+1}\right]$ that are not boundary arcs, there exists a sequence $x_{n} \in W^{q, \infty}(0, T)$ such that $x_{n}=0=x$ by (A6) on boundary $\operatorname{arcs}, x_{n}^{(j)}\left(\tau_{k}\right)=x^{(j)}\left(\tau_{k}\right)$ for all $j=0, \ldots, q-1$ and $k=0, \ldots, N+1$, and $x_{n} \rightarrow x$ in $H^{q}$. By (A3), we may define $v_{n} \in L^{\infty}(0, T)$ such that $D G(u) v_{n}=x_{n}$ for all $n$. It is readily seen that $v_{n} \in C(u)$ for all $n$ and $v_{n} \rightarrow v$ in $L^{2}$, which achieves the proof.

\section{Second-order Sufficient Conditions}

The second-order sufficient conditions theory classically involves two norms, namely $L^{2}$ and $L^{\infty}$, see Ioffe [10, Part III] and Maurer [20].

Assume that $X, Z$ are Banach spaces endowed the norms $\|\cdot\|_{X}$ and $\|\cdot\|_{Z}$, respectively, such that $Z \subset X$ with continuous embedding. Let $k \in \mathbb{N}$. We say that $r(x)=\mathcal{O}_{Z}\left(\|x\|_{X}^{k}\right)$ if $r(x) \leq C\|x\|_{X}^{k}$ for some $C>0$ when $\|x\|_{Z}$ is small enough. We say that $r(x)=o_{Z}\left(\|x\|_{X}^{k}\right)$ if $r(v) /\|x\|_{X}^{k}$ goes to zero when $\|x\|_{Z}$ goes to zero. In the sequel, $\|\cdot\|_{p}$ (resp. $\|\cdot\|_{r, p}$ ) denotes the norm of the space $L^{p}(0, T)$ (resp. the Sobolev space $W^{r, p}(0, T)$ ), for $1 \leq p \leq \infty$ and $r=1, \ldots<+\infty$. We write $\mathcal{O}_{p}$ and $\mathcal{O}_{r, p}$ for respectively $\mathcal{O}_{\|\cdot\|_{L} p}$ and $\mathcal{O}_{\|\cdot\|_{W^{r}, p}}$, and we use the same convention for $o_{p}$ and $o_{r, p}$. Similarly, $B_{p}$ and $B_{r, p}$ denote open balls in $L^{p}$ and $W^{r, p}$, respectively.

We remind that a quadratic form $Q(v)$ on a Hilbert space is a Legendre form (Ioffe and Tihomirov [11), if it is weakly lower semi-continuous (w.l.s.c.) and if $v_{n} \rightarrow v$ weakly and $Q\left(v_{n}\right) \rightarrow Q(v)$ strongly imply that $v_{n} \rightarrow v$ strongly.

The next theorem gives the second-order sufficient condition in its well-known form (i.e. without the curvature term).

Theorem 4.1. Let $u \in \mathcal{U}$ satisfy (19) with Lagrange multiplier $\eta$ and assume that (A2') holds. If the following second-order sufficient condition is satisfied:

$$
D_{u u}^{2} L(u, \eta)(v, v)>0 \quad \forall v \in C_{L^{2}}(u) \backslash\{0\}
$$

INRIA 
then $u$ is a local solution of (4) satisfying the quadratic growth condition (5).

Conversely, if (A1)-(A6) hold and if $\left(u, y_{u}\right)$ has no essential touch point (in particular, if the state constraint is of first order $q=1$ ), then the second-order sufficient condition (49) is satisfied iff the quadratic growth condition (5) is satisfied.

The proof of Theorem 4.1 will be given after a sequence of short lemmas.

Lemma 4.1. Let $(u, \eta) \in \mathcal{U} \times \mathcal{M}_{+}[0, T]$ and $v \in \mathcal{U}$. The following holds, for all $\sigma \in[0,1]$ :

$$
\begin{aligned}
\left\|y_{u+\sigma v}-y_{u}\right\|_{\infty} & =\mathcal{O}_{\infty}\left(\|v\|_{1}\right) \\
\left\|p_{u+\sigma v, \eta}-p_{u, \eta}\right\|_{\infty} & =\mathcal{O}_{\infty}\left(\|v\|_{1}\right) \\
\left\|z_{u+\sigma v, v}\right\|_{\infty} & =\mathcal{O}_{\infty}\left(\|v\|_{1}\right) \\
\left\|z_{u+\sigma v, v}-z_{u, v}\right\|_{\infty} & =\mathcal{O}_{\infty}\left(\|v\|_{2}^{2}\right) .
\end{aligned}
$$

Proof. Set $u_{\sigma}:=u+\sigma v$, and let $C$ denote a positive constant. Since $f$ is Lipschitz continuous by (A0), (50) is an easy consequence of Lemma A.1 Thus, $u_{\sigma}$ and $y_{u_{\sigma}}$ take values in a compact set of type:

$$
V_{\delta}=\left\{(\hat{u}, \hat{y}) \in \mathbb{R} \times \mathbb{R}^{n} ; \exists t \in[0, T],\left|(\hat{u}, \hat{y})-\left(u(t), y_{u}(t)\right)\right| \leq \delta\right\},
$$

for some $\delta>0$. The mappings $f, \ell$ and $g$ as well as their first order derivatives are $C^{1}$, and hence Lipschitz continuous over the compact set $V_{\delta}$. Lemma A.1 applied to the costate equation (7), ensures that $p_{u_{\sigma}, \eta}$ also remains uniformly bounded. The derivation of (51) and (52) being similar to the one of (53), we detail only the latter. We have (omitting time argument):

$$
\begin{aligned}
\left|\dot{z}_{u_{\sigma}, v}(t)-\dot{z}_{u, v}(t)\right| \leq & \left\|f_{y}\right\|_{\infty}\left|z_{u_{\sigma}, v}-z_{u, v}\right| \\
& +\left(\left|D f\left(u_{\sigma}, y_{u_{\sigma}}\right)-D f\left(u, y_{u}\right)\right|\right)\left(\left|z_{u, v}\right|+|v(t)|\right) .
\end{aligned}
$$

Since $D f$ is Lipschitz on $V$, we have by (50) $\left|D f\left(u_{\sigma}, y_{u_{\sigma}}\right)-D f\left(u, y_{u}\right)\right| \leq C\left(\|v\|_{1}+|v|\right)$. Combining with (52) and the inequality $a b \leq \frac{1}{2}\left(a^{2}+b^{2}\right)$, we deduce from the above display that

$$
\left|\dot{z}_{u_{\sigma}, v}(t)-\dot{z}_{u, v}(t)\right| \leq\left\|f_{y}\right\|_{\infty}\left|z_{u_{\sigma}, v}-z_{u, v}\right|+C\left(\|v\|_{1}^{2}+|v(t)|^{2}\right) .
$$

We conclude with Lemma A.1 and the inequality $\|v\|_{1} \leq \sqrt{T}\|v\|_{2}$.

Lemma 4.2. Let $(u, \eta) \in \mathcal{U} \times \mathcal{M}_{+}[0, T]$ and $v \in \mathcal{U}$. Then:

$$
L(u+v, \eta)=L(u, \eta)+D_{u} L(u, \eta) v+\frac{1}{2} D_{u u}^{2} L(u, \eta)(v, v)+r(v)
$$

with $r(v)=\mathcal{O}_{\infty}\left(\|v\|_{3}^{3}\right)$. In particular, $r(v)=o_{\infty}\left(\|v\|_{2}^{2}\right)$. 
Proof of Lemma [4.2 For $\sigma \in[0,1]$, set again $u_{\sigma}:=u+\sigma v$ and $p_{u_{\sigma}}:=p_{u_{\sigma}, \eta}$. By Lemma 2.1

$$
\begin{aligned}
r(v) & =\left[\int_{0}^{1}(1-\sigma)\left(D_{u u}^{2} L(u+\sigma v, \eta)-D_{u u}^{2} L(u, \eta)\right) \mathrm{d} \sigma\right](v, v) \\
& =\int_{0}^{1} \int_{0}^{T} \Delta_{1}(t) \mathrm{d} t \mathrm{~d} \sigma+\int_{0}^{1} \int_{0}^{T} \Delta_{2}(t) \mathrm{d} \eta(t) \mathrm{d} \sigma+\int_{0}^{1} \Delta_{3} \mathrm{~d} \sigma
\end{aligned}
$$

with (omitting time argument)

$$
\begin{aligned}
\Delta_{1}(t) & =D^{2} H_{(u, y)^{2}}\left(u_{\sigma}, y_{u_{\sigma}}, p_{u_{\sigma}}\right)\left(v, z_{u_{\sigma}, v}\right)^{2}-D^{2} H_{(u, y)^{2}}\left(u, y_{u}, p_{u}\right)\left(v, z_{u, v}\right)^{2} \\
\Delta_{2}(t) & =z_{u_{\sigma}, v}^{*} g_{y y}\left(y_{u_{\sigma}}\right) z_{u_{\sigma}, v}-z_{u, v}^{*} g_{y y}\left(y_{u}\right) z_{u, v} \\
\Delta_{3} & =z_{u_{\sigma}, v}(T)^{*} \phi_{y y}\left(y_{u_{\sigma}}(T)\right) z_{u_{\sigma}, v}(T)-z_{u, v}(T)^{*} \phi_{y y}\left(y_{u}(T)\right) z_{u, v}(T) .
\end{aligned}
$$

Under assumption (A0), second-order derivatives $g_{y y}$, etc. are Lipschitz continuous over a compact set $V_{\delta}$ defined in (54) for some $\delta>0$. By Lemma 4.1] we get, for some constant $C>0$ :

$$
\begin{aligned}
\Delta_{2}(t) & \leq C\left(\left|y_{u_{\sigma}}-y_{u}\right|\left|z_{u_{\sigma}, v}\right|^{2}+\left(\left|z_{u_{\sigma}, v}\right|+\left|z_{u, v}\right|\right)\left|z_{u_{\sigma}, v}-z_{u, v}\right|\right) \\
& \leq \mathcal{O}_{\infty}\left(\|v\|_{1}^{3}+\|v\|_{1}\|v\|_{2}^{2}\right) \leq \mathcal{O}_{\infty}\left(\|v\|_{3}^{3}\right),
\end{aligned}
$$

since by the Cauchy-Schwarz and Hölder inequalities, that give respectively

$$
\|\cdot\|_{2}^{2} \leq\|\cdot\|_{3}^{3 / 2}\|\cdot\|_{1}^{1 / 2} \quad ; \quad\|\cdot\|_{1} \leq T^{2 / 3}\|\cdot\|_{3},
$$

we have $\|\cdot\|_{2}^{2}\|\cdot\|_{1} \leq T\|\cdot\|_{3}^{3}$. Since the measure $\mathrm{d} \eta$ is bounded and the $\mathcal{O}_{\infty}$ are uniform w.r.t. time, we obtain $\int_{0}^{T} \Delta_{2}(t) \mathrm{d} \eta(t)=\mathcal{O}_{\infty}\left(\|v\|_{3}^{3}\right)$. The same upper bound holds for $\Delta_{3}(T)$. As for $\Delta_{1}(t)$, we have in the same way, by Lemma 4.1

$$
\begin{aligned}
\Delta_{1}(t) \leq & C\left(\left|y_{u_{\sigma}}-y_{u}\right|+\left|p_{u_{\sigma}}-p_{u}\right|+\sigma|v|\right)\left(\left|z_{u_{\sigma}, v}\right|^{2}+|v|^{2}\right) \\
& +C\left(\left|z_{u_{\sigma}, v}\right|+\left|z_{u, v}\right|+|v|\right)\left|z_{u_{\sigma}, v}-z_{u, v}\right| \\
\leq & C\left(\|v\|_{1}^{3}+\|v\|_{1}^{2}|v(t)|+\|v\|_{1}|v(t)|^{2}+|v(t)|^{3}\right) .
\end{aligned}
$$

Hence, $\int_{0}^{T} \Delta_{1}(t) \mathrm{d} t=\mathcal{O}_{\infty}\left(\|v\|_{3}^{3}\right)$. Finally, since the $\mathcal{O}_{\infty}$ do not depend on $\sigma \in[0,1]$, we obtain after integration over $[0,1]$ that $r(v)=\mathcal{O}_{\infty}\left(\|v\|_{3}^{3}\right)$. Since $\|\cdot\|_{3}^{3} \leq\|\cdot\|_{2}^{2}\|\cdot\|_{\infty}$, it follows that $r(v)=o_{\infty}\left(\|v\|_{2}^{2}\right)$.

Lemma 4.3. Let $(u, \eta) \in \mathcal{U} \times \mathcal{M}_{+}[0, T]$ satisfy (A2'). Then the quadratic form $\mathcal{U} \rightarrow \mathbb{R}$, $v \mapsto D_{u u}^{2} L(u, \eta)(v, v)$ has a unique extension to a continuous quadratic form over $L^{2}(0, T)$, and the latter is a Legendre form. 
Proof. Since $L^{\infty}$ is a dense subset of $L^{2}$ and $v \mapsto D_{u u}^{2} L(u, \eta)(v, v)$ is continuous for the norm of $L^{2}$, it has a unique continuous extension $Q$ over $L^{2}$. Set $p:=p_{u, \eta}$. By (10), we can write $Q(v)=Q_{0}(v)+Q_{1}(v)+Q_{2}(v)$ with:

$$
\begin{aligned}
Q_{2}(v)= & \int_{0}^{T} H_{y y}\left(u, y_{u}, p\right)\left(z_{u, v}, z_{u, v}\right) \mathrm{d} t \\
& +z_{u, v}(T)^{*} \phi_{y y}\left(y_{u}(T)\right) z_{u, v}(T)+\int_{0}^{T} z_{u, v}^{*} g_{y y}\left(y_{u}\right) z_{u, v} \mathrm{~d} \eta \\
Q_{1}(v)= & 2 \int_{0}^{T} H_{y u}\left(u, y_{u}, p\right)\left(z_{u, v}, v\right) \mathrm{d} t \\
Q_{0}(v)= & \int_{0}^{T} H_{u u}\left(u, y_{u}, p\right)(v, v) \mathrm{d} t
\end{aligned}
$$

Let $v_{n} \rightarrow \bar{v} \in L^{2}(0, T)$. The mapping $L^{2}(0, T) \mapsto H^{1}(0, T) ; v \mapsto z_{u, v}$ being linear continuous, $z_{n}:=z_{u, v_{n}}$ converges weakly to $\bar{z}:=z_{u, \bar{v}}$. Since $\left(z_{n}\right)$ is bounded in $H^{1}(0, T)$ and the inclusion of the latter in $C[0, T]$ is compact, $\left(z_{n}\right)$ is strongly convergent to $\bar{z}$, and thus $Q_{2}\left(v_{n}\right)$ converges strongly to $Q_{2}(\bar{v})$. The term $Q_{1}\left(v_{n}\right)$, bilinear in $\left(z_{n}, v_{n}\right)$, also converges strongly to $Q_{1}(\bar{v})$ when $z_{n}$ converges strongly and $v_{n}$ weakly. Therefore, $Q$ is a Legendre form iff $Q_{0}$ is one.

By (27), it is easily seen that $\gamma\|v\|_{2}^{2} \leq Q_{0}(v) \leq M\|v\|_{2}^{2}$, for some $M>0$. Since $H_{u u}\left(u(t), y_{u}(t), p(t)\right)$ is invertible at all $t$, the bilinear form associated with $Q_{0}$ induce a scalar product equivalent to the one of $L^{2}(0, T)$, in the sense that weak convergence for the usual scalar product of $L^{2}(0, T)$ and for the one of $Q_{0}$ are equivalent. Hence $Q_{0}$ has the same properties as $\|\cdot\|_{2}$, that is $Q_{0}$ is weakly l.s.c. and if $v_{n} \rightarrow \bar{v}$ in $L^{2}(0, T)$ and $Q_{0}\left(v_{n}\right) \rightarrow Q_{0}(\bar{v})$, then $v_{n} \rightarrow \bar{v}$. Consequently $Q_{0}$ and therefore $Q$ are Legendre forms, which achieves the proof.

Proof of Theorem [4.1] Assume that (49) holds but that the quadratic growth condition (5) is not satisfied. Then there exist a sequence $u_{n} \rightarrow u$ in $L^{\infty}, u_{n} \neq u$, such that $G\left(u_{n}\right) \in K$ for all $n$ and

$$
J\left(u_{n}\right) \leq J(u)+o\left(\left\|u_{n}-u\right\|_{2}^{2}\right) .
$$

Since $G\left(u_{n}\right) \in K$ and $\eta \in N_{K}(G(u))$, we have:

$$
J\left(u_{n}\right)-J(u)=L\left(u_{n}, \eta\right)-L(u, \eta)-\left\langle\eta, G\left(u_{n}\right)-G(u)\right\rangle \geq L\left(u_{n}, \eta\right)-L(u, \eta) .
$$

Since $u_{n}-u \rightarrow 0$ in $L^{\infty}$, Lemma 4.2 yields $r\left(u_{n}-u\right)=o\left(\left\|u_{n}-u\right\|_{2}^{2}\right)$. As $D_{u} L(u, \eta)=0$, we have:

$$
o\left(\left\|u_{n}-u\right\|_{2}^{2}\right) \geq J\left(u_{n}\right)-J(u) \geq \frac{1}{2} D_{u u}^{2} L(u, \eta)\left(u_{n}-u, u_{n}-u\right)+o\left(\left\|u_{n}-u\right\|_{2}^{2}\right) .
$$

Let $\left(v_{n}, \epsilon_{n}\right)$ be such that $u_{n}-u=\epsilon_{n} v_{n}$ with $\left\|v_{n}\right\|_{2}=1$ and $\epsilon_{n}=\left\|u_{n}-u\right\|_{2} \rightarrow 0$. Dividing by $\epsilon_{n}^{2}>0$ the above inequality, we get:

$$
D_{u u}^{2} L(u, \eta)\left(v_{n}, v_{n}\right)+o(1) \leq o(1) .
$$

The sequence $\left(v_{n}\right)$ being bounded in $L^{2}(0, T)$, taking if necessary a subsequence, we may assume that $\left(v_{n}\right)$ converges weakly to some $\bar{v} \in L^{2}(0, T)$. Since $D_{u u}^{2} L(u, \eta)$ is weakly l.s.c., we get passing to the limit:

$$
D_{u u}^{2} L(u, \eta)(\bar{v}, \bar{v}) \leq 0 .
$$


From (57), we derive that $J\left(u+\epsilon_{n} v_{n}\right)-J(u)=\epsilon_{n} D J(u) v_{n}+r_{n} \leq o\left(\epsilon_{n}^{2}\right)$, where $r_{n}=\mathcal{O}\left(\epsilon_{n}^{2}\right)$ (by the same arguments as in the proof of Lemma 4.2). Thus $D J(u) v_{n}+\mathcal{O}\left(\epsilon_{n}\right) \leq o\left(\epsilon_{n}\right)$, and passing to the limit, since the mapping $v \mapsto D J(u) v=\int_{0}^{T}\left(\ell_{y}\left(u, y_{u}\right) z_{u, v}+\ell_{u}\left(u, y_{u}\right) v\right) \mathrm{d} t+$ $\phi_{y}\left(y_{u}(T)\right) z_{u, v}(T)$ is weakly continuous, we obtain:

$$
D J(u) \bar{v} \leq 0 .
$$

Since $u+\epsilon_{n} v_{n} \in G^{-1}(K)$ (the inverse image of the set $\left.K\right), \bar{v}$ is a weak limit of $v_{n}=$ $\left(u_{n}-u\right) / \epsilon_{n} \in D G(u)^{-1} T_{K}(G(u))$. Since $K$ is closed and convex, so is $T_{K}(G(u))$. The mapping $D G(u)$ being continuous, $D G(u)^{-1} T_{K}(G(u))$ is also a closed convex set, and hence weakly closed. It follows that $\bar{v} \in D G(u)^{-1} T_{K}(G(u))$. Therefore, with [60,, $\bar{v} \in C_{L^{2}}(u)$. Thus (49) and (59) imply that $\bar{v}=0$. On the other hand, (58) gives (with $Q:=D_{u u}^{2} L(u, \eta)$ ):

$$
0=Q(\bar{v}) \leq \underline{\lim } Q\left(v_{n}\right) \leq \varlimsup \lim Q\left(v_{n}\right) \leq 0
$$

therefore $Q\left(v_{n}\right) \rightarrow Q(v)$. But $Q$ is a Legendre form by Lemma 4.3 and $v_{n} \rightarrow \bar{v}$, which implies that $v_{n} \rightarrow \bar{v}$ in $L^{2}(0, T)$, hence $\left\|v_{n}\right\|_{2} \rightarrow\|\bar{v}\|_{2}$. The expected contradiction arises since $\left\|v_{n}\right\|_{2}=1$ for all $n$ whereas $\|\bar{v}\|_{2}=0$.

The converse, that holds under stronger assumptions, is a consequence of Corollaries 3.13.2 For convenience, we prove it later with Theorem 5.1

\section{Reduction Approach}

There is still a gap between statements of Corollary 3.2 of Theorem 3.1 and Theorem 4.1 whenever essential touch points occur. We show in this section how to deal with this case, using a reduction approach in order to reformulate the constraint.

The idea of reductions methods (see e.g. 9]) is, when the constraint has finitely many contact points, to replace it by finitely many inequality constraints. The Hessian of Lagrangian of the corresponding reduced problem has an additional term that matches the curvature term. We obtain thus a no-gap second-order condition.

\subsection{General results on reduction}

It is known that the Sobolev spaces $W^{1, \infty}(0, T)$ and $W^{2, \infty}(0, T)$, endowed with the norms $\|x\|_{1, \infty}=\|x\|_{\infty}+\|\dot{x}\|_{\infty}$ and $\|x\|_{2, \infty}=\|x\|_{1, \infty}+\|\ddot{x}\|_{\infty}$, coincide with the spaces of Lipschitz continuous functions and the one of functions having a Lipschitz continuous derivative, respectively. For all $t, t_{0} \in[0, T], h \in W^{1, \infty}(0, T)$ and $x \in W^{2, \infty}(0, T)$, we have:

$$
\begin{aligned}
\left|h(t)-h\left(t_{0}\right)\right| & \leq\left|t-t_{0}\right|\|\dot{h}\|_{\infty}, \\
\left|x(t)-x\left(t_{0}\right)-\dot{x}\left(t_{0}\right)\left(t-t_{0}\right)\right| & \leq \frac{1}{2}\left|t-t_{0}\right|^{2}\|\ddot{x}\|_{\infty} .
\end{aligned}
$$

We now give some general results about zeros of functions of $W^{1, \infty}(0, T)$, and local minima/maxima of functions of $W^{2, \infty}(0, T)$. 
Lemma 5.1. Let $h_{0} \in W^{1, \infty}(0, T)$ and $\tau_{0} \in(0, T)$ satisfy the three following conditions: $h_{0}\left(\tau_{0}\right)=0 ; \dot{h}_{0}$ is continuous at $\tau_{0} ; \dot{h}_{0}\left(\tau_{0}\right) \neq 0$. Then for some $\delta, \varepsilon>0$, the mapping:

$$
\Xi: B_{1, \infty}\left(h_{0}, \delta\right) \mapsto\left(\tau_{0}-\varepsilon, \tau_{0}+\varepsilon\right) \quad ; \quad h \mapsto \tau_{h} \text { such that } h\left(\tau_{h}\right)=0,
$$

is well-defined and Lipschitz continuous on $B_{1, \infty}\left(h_{0}, \delta\right)$, and Fréchet differentiable at $h_{0}$, with derivative given by:

$$
D \Xi\left(h_{0}\right) d=-d\left(\tau_{0}\right) / \dot{h}_{0}\left(\tau_{0}\right), \quad \text { for all } d \in W^{1, \infty} .
$$

More precisely, we have for all $h, h_{i} \in B_{1, \infty}\left(h_{0}, \delta\right), i=1,2$ and $\tau_{i}=\tau_{h_{i}}$ :

$$
\begin{aligned}
\tau_{2}-\tau_{1} & =\mathcal{O}_{1, \infty}\left(\left\|h_{2}-h_{1}\right\|_{\infty}\right), \\
\dot{h}_{0}\left(\tau_{0}\right)\left(\tau_{h}-\tau_{0}\right)+h\left(\tau_{0}\right) & =o_{1, \infty}\left(\left\|h-h_{0}\right\|_{\infty}\right) .
\end{aligned}
$$

Proof. Assume w.l.o.g that $\beta:=\dot{h}_{0}\left(\tau_{0}\right)>0$, and denote by $c(\cdot)$ the modulus of continuity of $\dot{h}_{0}$ at $\tau_{0}$. Fix $\varepsilon>0$ such that $c(\varepsilon)<\frac{1}{4} \beta$. Thus, $\dot{h}_{0} \geq \frac{3}{4} \beta$ on $\left(\tau_{0}-\varepsilon, \tau_{0}+\varepsilon\right)$ and it follows that $h_{0}\left(\tau_{0}-\varepsilon\right)<-\frac{3}{4} \beta \varepsilon$ and $h_{0}\left(\tau_{0}+\varepsilon\right)>\frac{3}{4} \beta \varepsilon$. Set $\delta:=\min \left\{\frac{1}{4} \beta \varepsilon ; \frac{1}{4} \beta\right\}$ and let $h \in B_{1, \infty}\left(h_{0}, \delta\right)$. Thus, $h\left(\tau_{0}-\varepsilon\right)<0<h\left(\tau_{0}+\varepsilon\right)$ and $h$ is continuous, so $h$ has at least one zero $\tau_{h}$ in $\left(\tau_{0}-\varepsilon, \tau_{0}+\varepsilon\right)$. Let $\left(h_{1}, h_{2}\right) \in B_{1, \infty}\left(h_{0}, \delta\right)$ and $\tau_{i}$ such that $h_{i}\left(\tau_{i}\right)=0, i=1,2$. By the definition of $\delta$, we have $\dot{h}_{1} \geq \frac{1}{2} \beta$ a.e. on $\left(\tau_{0}-\varepsilon, \tau_{0}+\varepsilon\right)$, and, in consequence,

$$
\frac{\beta}{2}\left|\tau_{2}-\tau_{1}\right| \leq\left|h_{1}\left(\tau_{2}\right)\right|=\left|h_{1}\left(\tau_{2}\right)-h_{2}\left(\tau_{2}\right)\right| \leq\left\|h_{2}-h_{1}\right\|_{\infty} .
$$

Hence $\left|\tau_{2}-\tau_{1}\right| \leq \frac{2}{\beta}\left\|h_{2}-h_{1}\right\|_{\infty}$, which shows the uniqueness of the zero (take $h_{1}=h_{2}$ ), Lipschitz continuity and (65).

By continuity of $\Xi$ and $h_{0}$, and (61) applied to $h-h_{0}$, we have:

$$
\begin{aligned}
h_{0}\left(\tau_{h}\right)-\dot{h}_{0}\left(\tau_{0}\right)\left(\tau_{h}-\tau_{0}\right) & =o\left(\left|\tau_{h}-\tau_{0}\right|\right) \\
\left(h-h_{0}\right)\left(\tau_{h}\right)-\left(h-h_{0}\right)\left(\tau_{0}\right)=h_{0}\left(\tau_{h}\right)-h\left(\tau_{0}\right) & =\mathcal{O}\left(\left\|\dot{h}-\dot{h}_{0}\right\|_{\infty}\left|\tau_{h}-\tau_{0}\right|\right) .
\end{aligned}
$$

Since $\tau_{h}-\tau_{0}=\mathcal{O}_{1, \infty}\left(\left\|h-h_{0}\right\|_{\infty}\right)$ by (67), summing the above expansions yields (66), from which (64) follows.

Lemma 5.2. Let $x_{0} \in W^{2, \infty}(0, T)$ and $\tau_{0} \in(0, T)$ be such that $\dot{x}_{0}\left(\tau_{0}\right)=0, \ddot{x}_{0}$ is continuous at $\tau_{0}$ and $\ddot{x}_{0}\left(\tau_{0}\right)<0$. Thus $x_{0}$ has a local maximum at $\tau_{0}$, and for $\varepsilon>0$ and $\delta>0$ small enough, $x \in B_{2, \infty}\left(x_{0}, \delta\right)$ attains its maximum over $\left(\tau_{0}-\varepsilon, \tau_{0}+\varepsilon\right)$ at a unique point $\tau_{x}$. The mapping $\Theta: B_{2, \infty}\left(x_{0}, \delta\right) \rightarrow\left(\tau_{0}-\varepsilon, \tau_{0}+\varepsilon\right) ; x \mapsto \tau_{x}$ is Lipschitz continuous over $B_{2, \infty}\left(x_{0}, \delta\right)$, Fréchet differentiable at $x_{0}$, with derivative given by:

$$
D \Theta\left(x_{0}\right) w=-\dot{w}\left(\tau_{0}\right) / \ddot{x}_{0}\left(\tau_{0}\right) \quad \forall w \in W^{2, \infty} .
$$

Furthermore, the mapping

$$
\Phi: B_{2, \infty}\left(x_{0}, \delta\right) \rightarrow \mathbb{R} ; x \mapsto x\left(\tau_{x}\right),
$$


that associates with $x$ the value of its maximum on $\left(\tau_{0}-\varepsilon, \tau_{0}+\varepsilon\right)$, is $C^{1}$ over $B_{2, \infty}\left(x_{0}, \delta\right)$ and twice Fréchet differentiable at $x_{0}$ with first and second derivatives given by, for all $x \in B_{2, \infty}\left(x_{0}, \delta\right)$ and $d \in W^{2, \infty}$ :

$$
D \Phi(x) d=d\left(\tau_{x}\right) \quad ; \quad D^{2} \Phi\left(x_{0}\right)(d, d)=-\frac{\dot{d}\left(\tau_{0}\right)^{2}}{2 \ddot{x}_{0}\left(\tau_{0}\right)} .
$$

More precisely, for all $x, x_{i} \in B_{2, \infty}\left(x_{0}, \delta\right), i=1,2$ and $\tau_{i}=\tau_{x_{i}}$, we have:

$$
\begin{aligned}
x_{2}\left(\tau_{2}\right) & =x_{2}\left(\tau_{1}\right)+\mathcal{O}_{2, \infty}\left(\left\|x_{2}-x_{1}\right\|_{1, \infty}^{2}\right), \\
x\left(\tau_{x}\right) & =x\left(\tau_{0}\right)-\frac{\dot{x}\left(\tau_{0}\right)^{2}}{2 \ddot{x}_{0}\left(\tau_{0}\right)}+o_{2, \infty}\left(\left\|x-x_{0}\right\|_{1, \infty}^{2}\right) .
\end{aligned}
$$

Proof. Define $\delta$ as in the proof of Lemma [5.1] with $h_{0}$ replaced by $-\dot{x}_{0}$. It follows that for all $x \in B_{2, \infty}(x, \delta)$, there exists a unique $\tau_{x}$ satisfying $\dot{x}\left(\tau_{x}\right)=0$, and we have $\ddot{x}(t) \leq$ $\ddot{x}_{0}\left(\tau_{0}\right) / 2<0$ a.e. on $\left(\tau_{0}-\varepsilon, \tau_{0}+\varepsilon\right)$. Hence $\dot{x}$ is decreasing on $\left(\tau_{0}-\varepsilon, \tau_{0}+\varepsilon\right)$, and $x$ has unique maximum over $\left[\tau_{0}-\varepsilon, \tau_{0}+\varepsilon\right]$ attained at time $\tau_{x}$. By composition of the mapping $\Xi$ of Lemma 5.1 by the mapping $x \mapsto h=\dot{x} \in W^{1, \infty}, \Theta$ is well-defined, continuous over $B_{2, \infty}\left(x_{0}, \delta\right)$ and Fréchet differentiable at $x_{0}$, and (68) follows from (64).

By (62) applied to $x_{2}-x_{1}$, as $\dot{x}_{1}\left(\tau_{1}\right)=0$ and $\tau_{2}-\tau_{1}=\mathcal{O}_{2, \infty}\left(\left\|x_{2}-x_{1}\right\|_{1, \infty}\right)$ by (65), we get:

$$
\begin{aligned}
x_{2}\left(\tau_{2}\right) & =x_{2}\left(\tau_{1}\right)+\left(\dot{x}_{2}\left(\tau_{1}\right)-\dot{x}_{1}\left(\tau_{1}\right)\right)\left(\tau_{2}-\tau_{1}\right)+\mathcal{O}\left(\left|\tau_{2}-\tau_{1}\right|^{2}\right) \\
& =x_{2}\left(\tau_{1}\right)+\mathcal{O}_{2, \infty}\left(\left\|x_{2}-x_{1}\right\|_{1, \infty}^{2}\right)
\end{aligned}
$$

which shows (71) and proves that $\Phi$ is $C^{1}$ with first order derivative given by (70). By continuity of $\ddot{x}_{0}$ and (62) applied to $x-x_{0}$, we have, as $\dot{x}_{0}\left(\tau_{0}\right)=0$ :

$$
\begin{aligned}
x_{0}\left(\tau_{x}\right) & =x_{0}\left(\tau_{0}\right)+\ddot{x}_{0}\left(\tau_{0}\right) \frac{\left(\tau_{x}-\tau_{0}\right)^{2}}{2}+o\left(\left|\tau_{x}-\tau_{0}\right|^{2}\right), \\
\left(x-x_{0}\right)\left(\tau_{x}\right) & =\left(x-x_{0}\right)\left(\tau_{0}\right)+\dot{x}\left(\tau_{0}\right)\left(\tau_{x}-\tau_{0}\right)+\mathcal{O}\left(\left\|\ddot{x}-\ddot{x}_{0}\right\|_{\infty}\left|\tau_{x}-\tau_{0}\right|^{2}\right)
\end{aligned}
$$

Summing the above expansions, and since by (66),

$$
\tau_{x}-\tau_{0}=-\frac{\dot{x}\left(\tau_{0}\right)}{\ddot{x}_{0}\left(\tau_{0}\right)}+o_{2, \infty}\left(\left\|x-x_{0}\right\|_{1, \infty}\right),
$$

we obtain (72). Hence $\Phi$ is twice Fréchet differentiable at $x_{0}$ with second-order derivative given by (70).

\subsection{Application to optimal control problems.}

If the state constraint is of first order $q=1$, then Theorem 4.1 gives a no-gap second-order condition, that characterizes the quadratic growth. We show in this section how to extend 
this no-gap condition to the case when the trajectory has essential touch points (see Theorem 5.1).

Therefore, we assume in this section that the state constraint is not of first order, that is, the function $g^{(1)}(u, y)=g_{y}(y) f(u, y)$ does not depend on $u$ (which means $g_{u}^{(1)}(u, y) \equiv 0$ ). Note that this implies that $G(u)=g\left(y_{u}\right) \in W^{2, \infty}$, for all $u \in \mathcal{U}$.

Definition 5.1. Assume that $g_{u}^{(1)} \equiv 0$ (the state constraint is not of order one). Let $u \in G^{-1}(K)$. We say that a touch point $\tau$ of the trajectory $\left(u, y_{u}\right)$ is reducible, if the following conditions are satified: (i) the function $t \mapsto g^{(2)}\left(u(t), y_{u}(t)\right)$ is continuous at $\tau$; (ii) non-tangentiality condition (32) is satisfied at $\tau$.

Remark 5.1. 1) Point (i) in the above definition is always satisfied if the state constraint is of order $q>2$, since in that case $g^{(2)}\left(u, y_{u}\right)=g^{(2)}\left(y_{u}\right)$.

2) If $q=2$ and $\eta \in \Lambda(u) \neq \emptyset$, sufficient conditions for point (i) are assumptions (A2)-(A4), since by Prop. 2.11(i) they imply the continuity of $u$.

Let $u \in G^{-1}(\mathcal{U})$, and let $\mathcal{T}_{\text {red }}$ be a finite subset of reducible touch points of the trajectory $\left(u, y_{u}\right)$. By definition of touch points, there exists $\varepsilon>0$ such that $(\tau-2 \varepsilon, \tau+2 \varepsilon) \subset(0, T)$ and $(\tau-2 \varepsilon, \tau+2 \varepsilon) \cap I\left(g\left(y_{u}\right)\right)=\{\tau\}$, for all $\tau \in \mathcal{T}_{\text {red }}$. Set $I_{a}=\cup_{\tau \in \mathcal{T}_{\text {red }}}(\tau-\varepsilon, \tau+\varepsilon)$ and $I_{b}=[0, T] \backslash I_{a}$. Note that $I_{b}$ is closed. Let $N$ be the cardinal of $\mathcal{T}_{\text {red }}$ and denote by $\tau_{u}^{1}, \ldots, \tau_{u}^{N}$ the elements of $\mathcal{T}_{\text {red }}$. By definition of reducible touch points and continuity of the mapping $\mathcal{U} \mapsto W^{2, \infty}, u \mapsto g\left(y_{u}\right)$, we may apply Lemma [5.2. There exists $\delta>0$, such that for all $i=1, \ldots, N$, the mappings

$$
\mathcal{R}^{i}: B_{\infty}(\tilde{u}, \delta) \rightarrow \mathbb{R} \quad ; \quad \tilde{u} \mapsto g\left(y_{\tilde{u}}\left(\tau_{\tilde{u}}^{i}\right)\right),
$$

such that $g\left(y_{\tilde{u}}\right)$ attains its (unique) maximum over $\left[\tau_{u}^{i}-\varepsilon, \tau_{u}^{i}+\varepsilon\right]$ at time $\tau_{\tilde{u}}^{i}$, are well-defined. It follows that for all $\tilde{u} \in B_{\infty}(u, \delta)$,

$$
G(\tilde{u}) \in K \quad \text { iff } \quad g\left(y_{\tilde{u}}(t)\right) \leq 0 \forall t \in I_{b} \text { and } \mathcal{R}^{i}(\tilde{u}) \leq 0 \forall i=1, \ldots, N .
$$

Denote by $\left.g\left(y_{\tilde{u}}\right)\right|_{b}$ the restriction of $g\left(y_{\tilde{u}}\right)$ to $I_{b}$ and $\mathcal{R}: \tilde{u} \mapsto\left(\mathcal{R}^{i}(\tilde{u})\right)_{1 \leq i \leq N}$. The reduced problem is defined as follows:

$$
\min _{\tilde{u} \in \mathcal{B}_{\infty}(u, \delta)} J(\tilde{u}) \quad ; \quad \mathcal{G}(\tilde{u})=\left(\begin{array}{c}
\left.g(y \tilde{u})\right|_{b} \\
\mathcal{R}(\tilde{u})
\end{array}\right) \in \mathcal{K}:=C_{-}\left[I_{b}\right] \times \mathbb{R}_{-}^{N} .
$$

From (73), it follows that (74) is locally equivalent to problem (4) in a $L^{\infty}$ neighborhood of $u$. The Lagrangian $\mathcal{L}$ of the reduced problem (174) is given, for $\tilde{u} \in B_{\infty}(u, \delta)$ and $\lambda=$ $\left(\eta_{b}, \nu\right) \in \mathcal{M}_{+}\left[I_{b}\right] \times \mathbb{R}_{+}^{N}$, by:

$$
\mathcal{L}(\tilde{u}, \lambda)=J(\tilde{u})+\int_{I_{b}} g\left(y_{\tilde{u}}(t)\right) \mathrm{d} \eta_{b}(t)+\sum_{i=1}^{N} \nu_{i} \mathcal{R}^{i}(\tilde{u}) .
$$

The next lemma shows how the Lagrangian, multipliers and critical cone of the reduced problem (174) are related to the ones of problem (4). 
Lemma 5.3. Assume that $g_{u}^{(1)} \equiv 0$, and let $u \in G^{-1}(K)$ and $\mathcal{T}_{\text {red }}, I_{a}, I_{b}, \mathcal{R}, \mathcal{G}$ and $\mathcal{L}$ be defined as above. Let $\lambda=\left(\eta_{b}, \nu\right) \in \mathcal{M}_{+}\left[I_{b}\right] \times \mathbb{R}_{+}^{N}$. For $\delta>0$ small enough, the function $\tilde{u} \mapsto \mathcal{L}(\tilde{u}, \lambda)$ is $C^{1}$ on $B_{\infty}(u, \delta)$ and twice Fréchet differentiable at $u$. Define $\eta \in \mathcal{M}_{+}[0, T]$ by:

$$
\mathrm{d} \eta(t)=\mathrm{d} \eta_{b}(t) \text { on } I_{b} \quad ; \quad \mathrm{d} \eta(t)=\sum_{i=1}^{N} \nu_{i} \delta_{\tau_{u}^{i}}(t) \text { on } I_{a} .
$$

Then we have: $\mathcal{L}(u, \lambda)=L(u, \eta), D_{u} \mathcal{L}(u, \lambda)=D_{u} L(u, \eta)$,

$$
\begin{aligned}
& D \mathcal{G}(u)^{-1} T_{\mathcal{K}}(D \mathcal{G}(u))=D G(u)^{-1} T_{K}(G(u)), \\
& \lambda \in N_{\mathcal{K}}(\mathcal{G}(u)) \quad \text { iff } \quad \eta \in N_{K}(G(u)), \\
& D_{u u}^{2} \mathcal{L}(u, \lambda)=D_{u u}^{2} L(u, \eta)-\sum_{i=1}^{N} \nu_{i} \frac{\left(g_{y}^{(1)}\left(y_{u}\left(\tau_{u}^{i}\right)\right) z_{u, v}\left(\tau_{u}^{i}\right)\right)^{2}}{g^{(2)}\left(u\left(\tau_{u}^{i}\right), y_{u}\left(\tau_{u}^{i}\right)\right)} .
\end{aligned}
$$

Proof. Note that $\mathcal{R}^{i}=\Phi^{i} \circ G, i=1, \ldots, N$, where the mappings $\Phi^{i}$ are defined by (69) in Lemma 5.2 applied to $\left(x_{0}, \tau_{0}\right)=\left(g\left(y_{u}\right), \tau_{u}^{i}\right)$. It follows from Lemma 5.2 that $\mathcal{R}$ is $C^{1}$ over a small ball $B_{\infty}(u, \delta)$. By (70), the second-order expansion of the state (11) and (28) (since $\left.g_{u}^{(1)} \equiv 0\right)$, that gives $\frac{\mathrm{d}}{\mathrm{d} t} D G(u) v=g_{y}^{(1)}\left(y_{u}\right) z_{u, v}$, we see that, for all $v \in \mathcal{U}$ :

$$
\begin{gathered}
D \mathcal{R}^{i}(u) v=D \Phi^{i}(G(u)) D G(u) v=g_{y}\left(y_{u}\left(\tau_{u}^{i}\right)\right) z_{u, v}\left(\tau_{u}^{i}\right), \\
\begin{aligned}
& D^{2} \mathcal{R}^{i}(u)(v, v)= D \Phi^{i}(G(u)) D^{2} G(u)(v, v)+D^{2} \Phi^{i}(G(u))(D G(u) v, D G(u) v) \\
&= z_{u, v}\left(\tau_{u}^{i}\right)^{*} g_{y y}\left(y_{u}\left(\tau_{u}^{i}\right)\right) z_{u, v}\left(\tau_{u}^{i}\right)+g_{y}\left(y_{u}\left(\tau_{u}^{i}\right)\right) z_{u, v v}\left(\tau_{u}^{i}\right) \\
&-\frac{\left(g_{y}^{(1)}\left(y_{u}\left(\tau_{u}^{i}\right)\right) z_{u, v}\left(\tau_{u}^{i}\right)\right)^{2}}{g^{(2)}\left(u\left(\tau_{u}^{i}\right), y_{u}\left(\tau_{u}^{i}\right)\right)} .
\end{aligned}
\end{gathered}
$$

The conclusion follows easily from the above expressions (see the proof of Lemma 2.1), (77) is obtained as a consequence of (79).

It follows that if $u \in \mathcal{U}$ and $\Lambda(u) \neq \emptyset$, the Lagrange multipliers $\lambda$ and $\eta$ associated with $u$ in problems (74) and (4) respectively, are related by (76). By (77), it follows also that the critical cone $\mathcal{C}(u)$ for problem (74) is equal to $C(u)$. We shall show that the statement of Th. 4.1 remains true by replacing $L(u, \eta)$ by $\mathcal{L}(u, \lambda)$. That is, the main result of this paper, with Th. 3.1 (and Th. 4.1] for first-order state constraint), is the next theorem.

Theorem 5.1. Assume that $g_{u}^{(1)} \equiv 0$ (the state constraint is not of first order). Let $u \in \mathcal{U}$ satisfy (13) with Lagrange multiplier $\eta$, and assume that (A2') holds. Let $\mathcal{T}_{\text {red }}$ be a finite set of reducible touch points of $u$, and $\nu_{\tau}:=[\eta(\tau)]$. If the following second-order sufficient condition is satisfied:

$$
D_{u u}^{2} L(u, \eta)(v, v)-\sum_{\tau \in \mathcal{T}_{\text {red }}} \nu_{\tau} \frac{\left(g_{y}^{(1)}\left(y_{u}(\tau)\right) z_{u, v}(\tau)\right)^{2}}{\left.\frac{\mathrm{d}^{2}}{\mathrm{~d} t^{2}} g\left(y_{u}(t)\right)\right|_{t=\tau}}>0 \quad \forall v \in C_{L^{2}}(u) \backslash\{0\}
$$

INRIA 
then $u$ is a local solution of (4) satisfying the quadratic growth condition (5).

Conversely, if (A1)-(A6) hold, then the finitely many essential touch points of the trajectory $\left(u, y_{u}\right)$ are all reducible, and the second-order sufficient condition (80) is satisfied with $\mathcal{T}_{\text {red }}=\mathcal{T}_{\text {to }}^{\text {ess }}$ iff the quadratic growth condition (5) is satisfied.

Remark 5.2. Note that if $\mathcal{T}_{\text {red }}=\emptyset$, (80) coincides with (49). If $\mathcal{T}_{\text {red }}$ contains essential touch points, then by (32) the contribution in (80) of points in $\mathcal{T}_{\text {red }}$ is such that the sum is nonpositive, and therefore the sufficient condition (80) is in general weaker than (49).

We first need to extend Lemma 4.2 to the Lagrangian $\mathcal{L}$. Note that $\mathcal{L}$ is not $C^{2}$ in a $L^{\infty}$ neighborhood of $u$, thus (56) does not hold with $\mathcal{L}$.

Lemma 5.4. For $\delta>0$ small enough and all $v \in B_{\infty}(0, \delta)$,

$$
\mathcal{L}(u+v, \lambda)=\mathcal{L}(u, \lambda)+D_{u} \mathcal{L}(u, \lambda) v+\frac{1}{2} D_{u u}^{2} \mathcal{L}(u, \lambda)(v, v)+\tilde{r}(v)
$$

with $\tilde{r}(v)=o_{\infty}\left(\|v\|_{2}^{2}\right)$.

Proof. It is easily seen from (75) and (76) that

$$
\mathcal{L}(u+v, \lambda)=L(u+v, \eta)+\sum_{i=1}^{N} \nu_{i}\left(g\left(y_{u+v}\left(\tau_{u+v}^{i}\right)\right)-g\left(y_{u+v}\left(\tau_{u}^{i}\right)\right)\right) .
$$

We may write $\tilde{r}(v)=r(v)+\hat{r}(v)$, where $r(v)$ is given by (55) and satisfies $r(v)=\mathcal{O}\left(\|v\|_{3}^{3}\right)$ by Lemma 4.2. and by (78) we have $\hat{r}(v)=\sum_{i=1}^{N} \nu_{i} \hat{r}_{i}(v)$ with, for $i=1, \ldots, N$ :

$$
\hat{r}_{i}(v):=g\left(y_{u+v}\left(\tau_{u+v}^{i}\right)\right)-g\left(y_{u+v}\left(\tau_{u}^{i}\right)\right)+\frac{\left(g_{y}^{(1)}\left(y_{u}\left(\tau_{u}^{i}\right)\right) z_{u, v}\left(\tau_{u}^{i}\right)\right)^{2}}{2 g^{(2)}\left(u\left(\tau_{u}^{i}\right), y_{u}\left(\tau_{u}^{i}\right)\right)} .
$$

Fix $i=1, \ldots, N$, and set $x_{0}:=g\left(y_{u}\right)$ and $\tau_{0}:=\tau_{u}^{i}$. By definition of reducible touch points, $\left(x_{0}, \tau_{0}\right)$ satisfy the assumptions of Lemma [5.2] Set $x:=g\left(y_{u+v}\right) \in W^{2, \infty}$, then $\tau_{x}=\tau_{u+v}^{i}$, and since the state constraint is not of first order, we have $\dot{x}=g^{(1)}\left(y_{u+v}\right)$, $\ddot{x}=g^{(2)}\left(u+v, y_{u+v}\right)$ and hence, by (50):

$$
\left\|x-x_{0}\right\|_{1, \infty}=\mathcal{O}_{\infty}\left(\|v\|_{1}\right) \quad ; \quad\left\|\ddot{x}-\ddot{x}_{0}\right\|_{\infty}=\mathcal{O}_{\infty}\left(\|v\|_{\infty}\right) .
$$

Since $g^{(1)}\left(y_{u+v}\right)-g^{(1)}\left(y_{u}\right)-g_{y}^{(1)}\left(y_{u}\right) z_{u, v}=\int_{0}^{1}\left(g\left(y_{u+\sigma v}\right) z_{u+\sigma v}-g\left(y_{u}\right) z_{u}\right) \mathrm{d} \sigma$, we also have by (50) and (52)-(53), setting $h:=g_{y}^{(1)}\left(y_{u}\right) z_{u, v}$, that

$$
\left\|\dot{x}-\dot{x}_{0}-h\right\|_{\infty}=\mathcal{O}_{\infty}\left(\|v\|_{2}^{2}\right) .
$$

We may now write $\hat{r}_{i}(v)=\hat{r}_{i, 1}(v)+\hat{r}_{i, 2}(v)$ with:

$$
\hat{r}_{i, 1}(v)=x\left(\tau_{x}\right)-x\left(\tau_{0}\right)+\frac{\dot{x}\left(\tau_{0}\right)^{2}}{2 \ddot{x}_{0}\left(\tau_{0}\right)} \quad ; \quad \hat{r}_{i, 2}(v)=\frac{h\left(\tau_{0}\right)^{2}-\dot{x}\left(\tau_{0}\right)^{2}}{2 \ddot{x}_{0}\left(\tau_{0}\right)} .
$$


By (72) and (83), we have $\hat{r}_{i, 1}(v)=o_{\infty}\left(\|v\|_{1}^{2}\right)$. From $\left|a^{2}-b^{2}\right| \leq(2|a|+|a-b|)|a-b|$, $\|h\|_{\infty}=\mathcal{O}_{\infty}\left(\|v\|_{1}\right)$ by (152), (84) with $\dot{x}_{0}\left(\tau_{0}\right)=0$, and $\|\cdot\|_{2} \leq\|\cdot\|_{1}\|\cdot\|_{\infty}$, we see that $\hat{r}_{i, 2}(v)=$ $\mathcal{O}_{\infty}\left(\|v\|_{1}\|v\|_{2}^{2}\right) \leq \mathcal{O}_{\infty}\left(\|v\|_{1}^{2}\|v\|_{\infty}\right)$. It follows that $\hat{r}_{i}(v)=o_{\infty}\left(\|v\|_{1}^{2}\right)$ for all $i$ and finally that $\tilde{r}(v)=o_{\infty}\left(\|v\|_{2}^{2}\right)$, which achieves the proof.

Proof of Theorem 5.1. Since the sum of a Legendre form and of a weakly continuous quadratic form remains a Legendre form, we deduce easily from (75) and Lemma 4.3. since the additional terms

$$
v \mapsto z_{u, v}\left(\tau_{u}^{i}\right)^{*} \frac{g_{y}^{(1)}\left(y_{u}\left(\tau_{u}^{i}\right)\right)^{*} g_{y}^{(1)}\left(y_{u}\left(\tau_{u}^{i}\right)\right)}{g^{(2)}\left(u\left(\tau_{u}^{i}\right), y_{u}\left(\tau_{u}^{i}\right)\right)} z_{u, v}\left(\tau_{u}^{i}\right)
$$

are weakly continuous quadratic forms, that the unique continuous extension of $D_{u u} \mathcal{L}(u, \lambda)$ over $L^{2}$ is a Legendre form. In addition, since $\tilde{r}(v)=o_{\infty}\left(\|v\|_{2}^{2}\right)$ by Lemma [5.4 the proof of Theorem 4.1 still applies, replacing $L(u, \eta)$ by $\mathcal{L}(u, \lambda)$. It follows that (80) implies the quadratic growth condition (5).

Conversely, if (A1)-(A6) hold, there are finitely many essential touch points of $\left(u, y_{u}\right)$, all being reducible. Assume that (5) holds. Then for sufficiently small $\varepsilon>0, u$ is solution of the following problem:

$$
\left(\mathcal{P}_{\varepsilon}\right) \quad \min _{\tilde{u} \in L^{2}}\left\{J^{\varepsilon}(\tilde{u}):=J(\tilde{u})-\varepsilon\|\tilde{u}-u\|_{2}^{2}\right\} \quad ; \quad G(\tilde{u}) \in K,
$$

with the same Lagrange multiplier $\eta$ as $D_{u} J^{\varepsilon}(u)=D_{u} J(u)$. Since in addition $\left(\mathcal{P}_{\varepsilon}\right)$ and (4) have the same constraints, they have the same critical cone. Denote the Lagrangian of $\left(\mathcal{P}_{\varepsilon}\right)$ by $L^{\varepsilon}(u, \eta)$. Note that if assumptions (A0)-(A6) are satisfied for problem (4), so are they for problem [85], so that, for $\varepsilon$ small enough, $H_{u u}^{\varepsilon}=H_{u u}-\varepsilon$ is positive, uniformly over $t$. The extended second-order necessary condition in Corollary 3.2 for $\left(\mathcal{P}_{\varepsilon}\right)$ yields, since $D_{u u}^{2} L^{\varepsilon}(u, \eta)(v, v)=D_{u u}^{2} L(u, \eta)(v, v)-\varepsilon\|v\|_{2}^{2}$ :

$$
D_{u u}^{2} L(u, \eta)(v, v)-\sum_{\tau \in \mathcal{T}_{\text {to }}^{\text {ess }}} \nu_{\tau} \frac{\left(g_{y}^{(1)}\left(y_{u}(\tau)\right) z_{u, v}(\tau)\right)^{2}}{\left.\frac{\mathrm{d}^{2}}{\mathrm{~d} t^{2}} g\left(y_{u}(t)\right)\right|_{t=\tau}} \geq \varepsilon\|v\|_{2}^{2}, \quad \forall v \in C_{L^{2}}(u) .
$$

Hence 80 is satisfied with $\mathcal{T}_{\text {red }}=\mathcal{T}_{\text {to }}^{\text {ess }}$.

Note that taking $\mathcal{T}_{\text {red }}=\emptyset=\mathcal{T}_{\text {to }}^{\text {ess }}$ proves the converse in Th. 4.11, when $\left(u, y_{u}\right)$ has no essential touch point (including the case $q=1$ ).

Remark 5.3. The second-order sufficient condition in (80) remains in quite an abstract form, of little help to check the optimality of a trajectory in application to real life problems. Some verifiable second-order sufficient conditions exist in the literature that are based on Ricatti equations, see e.g. [20]. They may be too strong, however, since they ensure in general the coercivity of the Hessian of the Lagrangian over a space that is larger than the critical cone $C_{L^{2}}(u)$. See also [17, 18] for first order state constraints.

Remark 5.4. Handling an infinite number of junction points remains an open problem. It was shown indeed by Robbins in [23, on an example involving a third order state constraint, 
and though satisfying all regularity assumptions (A0)-(A3), that the optimal trajectory has a boundary arc, but except for a nowhere dense subset of initial conditions $y_{0}$, the latter is not regular, its entry point being the cluster point of an infinite sequence of touch points.

\section{Conclusion}

Our main result is a no-gap condition for an optimal control problem with a single state constraint of any order and only one control. The main hypotheses are that there are finitely many junction points, the essential touch points being reducible, the entry/exit points being regular, and strict complementarity on boundary arcs. It should not be too difficult to deal with the results in the case when $g\left(y_{u}(T)\right)=0$.

An open problem of interest is the characterization of strong regularity in the sense of Robinson [26] for optimal control problems with state constraints. See related results in [4] Section 5.1] and Malanowski [16].

We hope in the future to extend the results of this paper to the case of several state constraints and control variables, and to relate these second-order conditions to the study of the well-posedness of the shooting algorithm.

\section{A Appendix}

Lemma A.1 (Extension of Gronwall Lemma). Let $p \in B V\left([0, T] ; \mathbb{R}^{n}\right)$ be such that:

$$
|\mathrm{d} p(t)| \leq \kappa|p(t)| \mathrm{d} t+\mathrm{d} \mu(t), \quad \forall t \in[0, T],
$$

for some positive constant $\kappa$, and a nonnegative bounded measure $\mu$. Then:

$$
\|p\|_{\infty} \leq e^{\kappa T}|p(0)|+\int_{0}^{T} e^{\kappa(T-t)} \mathrm{d} \mu(t) .
$$

Proof. Set $\rho(t)=|p(t)|$. Then $\rho$ is a nonnegative bounded measure, and for all $t \in[0, T)$ and $s \rightarrow 0^{+}$, we have:

$$
\begin{aligned}
\int_{t}^{t+s} \mathrm{~d} \rho(\sigma) & =\rho(t+s)-\rho(t)=|p(t+s)|-|p(t)| \\
& \leq|p(t+s)-p(t)|=\left|\int_{t}^{t+s} \mathrm{~d} p(\sigma)\right| \leq \int_{t}^{t+s}|\mathrm{~d} p(\sigma)| .
\end{aligned}
$$

From (87) it follows that $\rho(t) \leq \varphi(t)$ for all $t \in[0, T]$, where $\varphi$ is solution of

$$
\varphi(t)=|p(0)|+\kappa \int_{0}^{t} \varphi(s) \mathrm{d} s+\int_{0}^{t} \mathrm{~d} \mu(s), \quad \text { for all } t \in[0, T] .
$$

Then

$$
\mathrm{d}\left(e^{-\kappa t} \varphi(t)\right)=e^{-\kappa t} \mathrm{~d} \varphi(t)-\kappa e^{-\kappa t} \varphi(t) \mathrm{d} t=e^{-\kappa t} \mathrm{~d} \mu(t) .
$$

Therefore, $e^{-\kappa t} \rho(t)=|p(0)|+\int_{0}^{t} e^{-\kappa s} \mathrm{~d} \mu(s)$. The result follows. 
Lemma A.2 (Integration by parts). For any $p \in B V\left([0, T], \mathbb{R}^{n *}\right)$ and $z \in W^{1,1}\left(0, T ; \mathbb{R}^{n}\right)$, the following relation holds:

$$
\int_{0}^{T} \mathrm{~d} p(t) z(t)=-\int_{0}^{T} p(t) \dot{z}(t) \mathrm{d} t+p(T) z(T)-p(0) z(0) .
$$

Proof. It is sufficient to give the proof when $n=1$. Since $p$ has bounded variation, it has at most countably many discontinuity times. Assume first that $p$ has finitely many discontinuity times $0 \leq \tau_{1}<\ldots<\tau_{N} \leq T$. Set $\tau_{0}:=0$ and $\tau_{N+1}:=T$. We have:

$$
\int_{0}^{T} \mathrm{~d} p(t) z(t)=\sum_{n=1}^{N+1} \int_{\tau_{n-1}}^{\tau_{n}} \dot{p}(t) z(t) \mathrm{d} t+\sum_{n=1}^{N}\left[p\left(\tau_{n}\right)\right] z\left(\tau_{n}\right)
$$

Since $p$ and $z$ are absolutely continuous on $\left(\tau_{n-1}, \tau_{n}\right)$, we may integrate by parts:

$$
\begin{aligned}
\int_{0}^{T} \mathrm{~d} p(t) z(t)= & \sum_{n=1}^{N+1} \int_{\tau_{n-1}}^{\tau_{n}}-p(t) \dot{z}(t) \mathrm{d} t-\sum_{n=1}^{N+1} p\left(\tau_{n-1}^{+}\right) z\left(\tau_{n-1}\right) \\
& +\sum_{n=1}^{N+1} p\left(\tau_{n}^{-}\right) z\left(\tau_{n}\right)+\sum_{n=1}^{N}\left[p\left(\tau_{n}\right)\right] z\left(\tau_{n}\right),
\end{aligned}
$$

which immediately gives [88. Assume now that $p$ has countably many discontinuity times, $\left(\tau_{n}\right), n \in \mathbb{N}$, (the latter being not necessarily in increasing order) with jumps discontinuities $\nu_{n}=\left[p\left(\tau_{n}\right)\right]$, such that $\sum_{n \in \mathbb{N}}\left|\nu_{n}\right| \leq\|\mathrm{d} p\|<+\infty$. Denote by $p_{a}$ the absolutely continuous part of $p$, i.e. such that $\mathrm{d} p=p_{a} \mathrm{~d} t+\sum_{n \in \mathbb{N}} \nu_{n} \delta_{\tau_{n}}$. For $N>0$, set $\mathrm{d} p_{N}:=p_{a} \mathrm{~d} t+\sum_{n=0}^{N} \nu_{n} \delta_{\tau_{n}}$. It follows that, for all $N$,

$$
\int_{0}^{T} \mathrm{~d} p_{N}(t) z(t)=-\int_{0}^{T} p_{N}(t) \dot{z}(t) \mathrm{d} t+p_{N}(T) z(T)-p_{N}(0) z(0) .
$$

Since we have

$$
\left|\mathrm{d}\left(p-p_{N}\right)(t)\right| \leq \sum_{n=N+1}^{\infty}\left|\nu_{n}\right| \delta_{\tau_{n}}(t)
$$

by Lemma A.1 we deduce that $\left\|p-p_{N}\right\|_{\infty} \leq \sum_{n=N+1}^{\infty}\left|\nu_{n}\right| \rightarrow 0$ when $N \rightarrow+\infty$. Hence we can pass to the limit in [89], which gives the result.

\section{References}

[1] J.F. Bonnans. Second order analysis for control constrained optimal control problems of semilinear elliptic systems. Journal of Applied Mathematics \& Optimization, 38:303$325,1998$. 
[2] J.F. Bonnans, R. Cominetti, and A. Shapiro. Sensitivity analysis of optimization problems under abstract constraints. Mathematics of Operations Research, 23:806-831, 1998.

[3] J.F. Bonnans, R. Cominetti, and A. Shapiro. Second order optimality conditions based on parabolic second order tangent sets. SIAM Journal on Optimization, 9:466-492, 1999.

[4] J.F. Bonnans and A. Shapiro. Perturbation analysis of optimization problems. SpringerVerlag, New York, 2000.

[5] A.E. Bryson, W.F. Denham, and S.E. Dreyfus. Optimal programming problems with inequality constraints I: necessary conditions for extremal solutions. AIAA Journal, 1:2544-2550, 1963.

[6] R. Cominetti. Metric regularity, tangent sets and second order optimality conditions. Journal of Applied Mathematics \&3 Optimization, 21:265-287, 1990.

[7] R. Cominetti and J.P. Penot. Tangent sets to unilateral convex sets. Comptes Rendus de l'Académie des Sciences de Paris, Série I, 321:1631-1636, 1995.

[8] A. Haraux. How to differentiate the projection on a convex set in Hilbert space. Some applications to variational inequalities. Journal Mathematical Society of Japan, 29:615$631,1977$.

[9] R.P. Hettich and H.T. Jongen. Semi-infinite programming: conditions of optimality and applications. In J. Stoer, editor, Optimization Techniques. Proc. 8th IFIP Conf. on Optimization Techniques, Würzburg. Part 2, Springer-Verlag, New York, 1978.

[10] A.D. Ioffe. Necessary and sufficient conditions for a local minimum I: A reduction theorem and first order conditions, II: Conditions of Levitin-Miljutin-Osmolovskii type, III: Second order conditions and augmented duality. SIAM Journal on Control Optimization, 17:245-250, 251-265 and 266-288, 1979.

[11] A.D. Ioffe and V.M. Tihomirov. Theory of Extremal Problems. North-Holland Publishing Company, Amsterdam, 1979. Russian Edition: Nauka, Moscow, 1974.

[12] D.H. Jacobson, M.M. Lele, and J.L. Speyer. New necessary conditions of optimality for control problems with state-variable inequality contraints. J. of Mathematical Analysis and Applications, 35:255-284, 1971.

[13] H. Kawasaki. An envelope-like effect of infinitely many inequality constraints on second order necessary conditions for minimization problems. Mathematical Programming, 41:73-96, 1988.

[14] H. Kawasaki. The upper and lower second order directional derivatives of a sup-type function. Mathematical Programming, 41:327-339, 1988. 
[15] H. Kawasaki. Second order necessary optimality conditions for minimizing a sup-type function. Mathematical Programming (Ser. A), 49:213-229, 1990/91.

[16] K. Malanowski. Second order conditions and constraint qualifications in stability and sensitivity analysis of solutions to optimization problems in Hilbert spaces. Journal of Applied Mathematics \& Optimization, 25:51-79, 1992.

[17] K. Malanowski. Sufficient optimality conditions for optimal control subject to state constraints. SIAM J. on Control and Optimization, 35:205-227, 1997.

[18] K. Malanowski, H. Maurer, and S. Pickenhain. Second-order sufficient conditions for state-constrained optimal control problems. J. of Optimization Theory and Applications, 123:595-617, 2004.

[19] H. Maurer. On the minimum principle for optimal control problems with state constraints. Schriftenreihe des Rechenzentrum 41, Universität Münster, 1979.

[20] H. Maurer. First and second order sufficient optimality conditions in mathematical programming and optimal control. Math. Programming Stud., (14):163-177, 1981.

[21] H. Maurer and J. Zowe. First and second-order necessary and sufficient optimality conditions for infinite-dimensional programming problems. Mathematical Programming, 16:98-110, 1979.

[22] F. Mignot. Contrôle dans les inéquations variationnelles elliptiques. Journal of Functional Analysis, 22:130-185, 1976.

[23] H.M. Robbins. Junction phenomena for optimal control with state-variable inequality constraints of third order. J. of Optimization Theory and Applications, 31:85-99, 1980.

[24] S.M. Robinson. First order conditions for general nonlinear optimization. SIAM Journal on Applied Mathematics, 30:597-607, 1976.

[25] S.M. Robinson. Stability theorems for systems of inequalities, part II: Differentiable nonlinear systems. SIAM Journal on Numerical Analysis, 13:497-513, 1976.

[26] S.M. Robinson. Strongly regular generalized equations. Mathematics of Operations Research, 5:43-62, 1980.

[27] J. Sokolowski. Sensitivity analysis of control constrained optimal control problems for distributed parameter systems. SIAM Journal on Control and Optimization, 25:1542$1556,1987$.

[28] V. Zeidan. The Riccati equation for optimal control problems with mixed state-control constraints: necessity and sufficiency. SIAM J. on Control and Optimization, 32:1297$1321,1994$. 


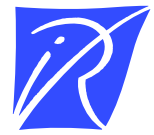

Unité de recherche INRIA Rocquencourt

Domaine de Voluceau - Rocquencourt - BP 105 - 78153 Le Chesnay Cedex (France)

Unité de recherche INRIA Futurs : Parc Club Orsay Université - ZAC des Vignes

4, rue Jacques Monod - 91893 ORSAY Cedex (France)

Unité de recherche INRIA Lorraine : LORIA, Technopôle de Nancy-Brabois - Campus scientifique

615, rue du Jardin Botanique - BP 101 - 54602 Villers-lès-Nancy Cedex (France)

Unité de recherche INRIA Rennes : IRISA, Campus universitaire de Beaulieu - 35042 Rennes Cedex (France)

Unité de recherche INRIA Rhône-Alpes : 655, avenue de l'Europe - 38334 Montbonnot Saint-Ismier (France)

Unité de recherche INRIA Sophia Antipolis : 2004, route des Lucioles - BP 93 - 06902 Sophia Antipolis Cedex (France) 